\title{
Synchronization of bidirection multiple neural networks with impulsive coupling control
}

\author{
Yong Wang ${ }^{1}$, Ailong $\mathrm{Wu}^{2 *}$ and Biwen $\mathrm{Li}^{2}$
}

"Correspondence:
hbnuwu@yeah.net
${ }^{2}$ College of Mathematics and
Statistics, Hubei Normal University,
Huangshi, 435002, China
Full list of author information is
available at the end of the article

available at the end of the article

\begin{abstract}
This paper is devoted to the study of the global synchronization of multiple neural networks (MNNs) via impulsive coupling control. Compared to one-layer neural networks, MNNs have more complex dynamics behavior and greater practical significance. In this paper, two interconnected bidirection MNNs are constructed; for this type of neural network system, each node in their network will be affected by the corresponding node in another network, then the synchronization problem of unidirection MNNs is extended to bidirection MNNs. In order to achieve synchronization of the bidirection MNNs, we adopt the method of impulsive coupling control, and together with the appropriate Lyapunov function, we deduce the relationship of the synchronization error system at the adjacent two impulsive moments, through iteration, and we finally obtain sufficient conditions to realize the global synchronization of the two interconnected bidirection MNNs. We illustrate the effectiveness of the results by simulation.
\end{abstract}

Keywords: Multiple neural networks; Impulsive coupling control; Synchronization; Coupling strength; Variable topology

\section{Introduction}

As an important subject, neural networks (NNs) have attracted more and more attention of scholars. Because of their superior performance, they have been widely used in associative memory, signal processing, optimization, machine learning and so on. Common dynamic behaviors just like periodicity, synchronization, chaos, multi-stability, dissipation, stability, self-association and other dynamic behaviors have been widely studied, and new results are still emerging (see [1-3]).

As an important dynamic behavior in neural network, synchronization is of great significance [4]. At present, there are many excellent research results in neural network synchronization. Ramirez et al. [5] study master-slave synchronization, and propose potential solutions to the problem that master-slave synchronization control scheme cannot induce synchronization. In Ref. [6], a new impulsive pinning strategy involving pinning ratio has been proposed to study pinning synchronization under impulsive control. In Ref. [7], Fečkan et al. study the synchronization of a nonlinear fractional system, and ana-

(c) The Author(s) 2020. This article is licensed under a Creative Commons Attribution 4.0 International License, which permits use, sharing, adaptation, distribution and reproduction in any medium or format, as long as you give appropriate credit to the original author(s) and the source, provide a link to the Creative Commons licence, and indicate if changes were made. The images or other third party material in this article are included in the article's Creative Commons licence, unless indicated otherwise in a credit line to the material. If material is not included in the article's Creative Commons licence and your intended use is not permitted by statutory regulation or exceeds the permitted use, you will need to obtain permission directly from the copyright holder. To view a copy of this licence, visit http://creativecommons.org/licenses/by/4.0/. 
lyze the time response and chaotic behaviors of this system. Alsaedi et al. [8] have studied the finite-time synchronization of a class of complex dynamical networks with Markovian hopping and time-varying delay. Generalized multi-synchronization is studied in Ref. [9]. Cruz-Ancona et al. [10] study the problem of generalized multi-synchronization in a master-slave topology in the framework of differential algebra and design a control protocol that can solve the problem of leadership following consensus. A class of sampling-data synchronization is given in Ref. [11], which is through the method of sampling-data synchronization control to make the system with time-varying sampling period achieve synchronization, the sampling period in [11] is time-varying, switching between two values according to random probability, it is a synchronous way which is defined by the characteristics of the controller.

The dynamic behavior of multiple neural networks (MNNs), which is composed of multiple sub-networks, has been more and more concerned in the past 20 years, and the common examples are the robot group [12], the unmanned aerial vehicle group, and the migration of fish stocks. The synchronization behavior of MNNs is embodied as a common behavior for each sub-network with different initial state. In the multi-network synchronization scheme, the coupling between each subnetwork has great effects, resulting in synchronization under various coupling schemes, such as linear coupling. Lu and Chen [13] use variational methods to study the local synchronization of linear coupled NNs, which is also an earlier study of coupled networks. Liu and Liao [14] study a class of finite-time driven-response synchronization problems for a general delay memory neural network (DMNN) with interval parameters, fuzzy logic system models, and nonlinear coupling, and they present a unified condition for finite-time synchronization of a fuzzy DMNN with nonlinear coupling. As for fixed topology coupling, Wang and Cao [15] study the exponential synchronization of a class of coupled NNs with probabilistic time-varying delay coupling and time-varying impulsive delay based on random memory. Mathiyalagan et al. [16] study the impulsive synchronization of a class of BAM NNs based on time-varying delay. Li and Cao [17] study the synchronization of inertial memristor system with linear coupling, and use a variety of methods, such as differential inclusion method and matrix measure method, the coupling topology is also fixed. In practical applications, some links are broken, or new links are generated; then the coupling topology is not always fixed, for instance, in limited communication channel, links are usually changed as needed to reduce redundant connections and save resources, so a concept of switching topology is proposed to describe the sudden change of the connection topology of NNs. Because the study of switched topology meets the needs of network change in practical applications, it motivates many scholars. In NNs with switching topology, synchronization is one of the dynamics we have to study. In this regard, Yang et al. [18] study a class of multi-recursive NNs with impulsive coupling control and give sufficient conditions for global synchronization in which the coupling topology is variable and introduce the concepts of sequential connection and common connection to describe the switching mode of coupling topology.

In many studies, the coupling scheme has always been effective, but we may only need coupling in some times, and the communication bandwidth is limited, so this kind of coupling scheme causes a lot of unnecessary cost waste. Therefore, if the coupling between NNs only occurs at some discrete time (such as impulsive time), then the cost of the application can be effectively reduced, so it is necessary to study the impulsive coupling control scheme. In [18-21], the impulsive coupling control is used to switch the coupling topol- 
ogy at the impulsive time, so as to achieve better control effect and practical significance. In the non-impulsive time, each node is independent, and information exchange only occurs at the impulsive time, which greatly reduces the waste of communication resources. There are examples of this in real life, for example, a synchronous flash of fireflies, the flash of each firefly in a population as an impulsive coupled signal, which finally achieves a synchronous flash.

The structure of the NNs is complex and diverse. For bidirectional NNs, as early as 1987, a bidirectional associative memory (BAM) NNs with bilayer is first proposed by Kosko. What is special about this type of neural network is that its u-layer is not independent of the v-layer, but is closely linked through the respective neurons. Because of this particularity, this type of NNs has been used in the fields of artificial intelligence, signal processing, combination optimization and image processing. For this network model, in [22], Cheng et al. investigate the Mittag-Lefflfler synchronization and stability of fractional-order BAM NNs. Similarly, for this characteristic of the BAM NNs, if we apply it on the MNNs, then we can combine the characteristics of the two networks, which is also very meaningful.

In practice, it is inevitable to study the NNs with time-delay, because of the limited switching speed of the amplifier and the limited information processing speed, thus a large amount of complex dynamical behaviors can be generated. And in the neural network model study, if the type or size of the time-delay is different, then the results may also be completely different. The common delays in currently studies are discrete delay, time-varying delay, state-dependent state delay, and infinite time-delay [23].

According to the above discussion, in this paper, we study the synchronization problem of bidirectional MNNs with time-delay under impulsive coupling control. Through the impulsive coupling controller, we finally achieve the synchronization of the two MNNs. The coupling controller in this paper makes the two networks to be coupled only at the discrete time, the coupling topology will switch with time, and it is sequential connection. The two MNNs are not only affected by their respective impulsive coupling controllers, but also by the corresponding nodes of another network. Generally speaking, the main work of this paper can be summed up in the following two points:

(1) The synchronization problem of two interconnected bidirection MNNs with impulsive coupling control is studied. Because impulsive control is adopted, communication resources can be saved. In addition, the design of impulsive coupling controller is more convenient through coupling intensity selection.

(2) We give the sufficient conditions of MNNs with time-delay to achieve global synchronization under impulsive coupling control.

The remaining part of this paper is organized as follows. Section 2 gives some preliminaries on graph theory, the system of bidirection MNNs and impulsive coupling controller. Section 3 derives sufficient conditions for global synchronization of the continuous-time MNNs with impulsive interactions. Section 4 gives a numerical example to substantiate the theoretical analysis. Finally, the conclusion of this paper is given in Sect. 5.

\section{Preliminaries}

\subsection{Graph theory}

A graph $\mathrm{G}=(\mathrm{V}, \mathrm{E})$ is composed of two sets: $\mathrm{V}$ is the set of nodes, and $\mathrm{E} \subseteq \mathrm{V} \times \mathrm{V}$ is the set of edges. Each graph G $=(\mathrm{V}, \mathrm{E})$ has a unique nonnegative matrix $A=\left(a_{i j}\right)_{N \times N} \in R^{N \times N}$ corresponding to it where $a_{i j}>0$ represents the connection relationship between node $i$ 
and node $j,(i, j) \in \mathrm{V} \times \mathrm{V}$. The union of two graphs $\mathrm{G}_{1}=\left(\mathrm{V}, \mathrm{E}_{1}\right)$ and $\mathrm{G}_{2}=\left(\mathrm{V}, \mathrm{E}_{2}\right)$ is defined by $\mathrm{G}_{1} \cup \mathrm{G}_{2}=\left(\mathrm{V}, \mathrm{E}_{1} \cup \mathrm{E}_{2}\right)$.

Given a graph $\mathrm{G}=(\mathrm{V}, \mathrm{E})$ and a nonempty subset $\mathrm{N} \subseteq \mathrm{V}$, the neighbors of $\mathrm{N}$ are defined as the set $\mathrm{M}(\mathrm{N}, \mathrm{G})=\{j \in \mathrm{V} \backslash \mathrm{N} \mid \exists i \in \mathrm{N}$, such that $(i, j) \in \mathrm{E}\}$. If the set $\mathrm{N}$ is a singleton, then $\mathrm{M}(\mathrm{N}, \mathrm{G})$ represents the neighbor of one node.

If $\bigcup_{i=1}^{m} \mathrm{G}_{i}$ contains a spanning tree (a detailed introduction is provided in [19]), then the sequence of graphs $\left(\mathrm{G}_{i}\right)_{i=1}^{m}$ is jointly connected. If the set of $\mathrm{V}_{k}$ satisfies $\mathrm{V}_{k} \subseteq \mathrm{V}(1 \leq k \leq$ $m+1)$ and $\mathrm{V}_{k+1} \subseteq \mathrm{V}_{k} \cup \mathrm{N}\left(\mathrm{G}_{k}, \mathrm{~V}_{k}\right)$ where $\mathrm{V}_{1}$ is a singleton, $\mathrm{V}_{m+1}=\mathrm{V}$, then the sequence of graphs $\left(\mathrm{G}_{i}\right)_{i=1}^{m}$ is sequentially connected. It is also T-sequential connected with the period $T=m$. For some more properties of jointly connected and sequentially connected cases refer to $[18,19]$.

Remark 2.1 Note that each graph of $\mathrm{G}_{i}$ has the common nodes. In addition, we can see that, if the sequence of graphs $\left(\mathrm{G}_{i}\right)_{i=1}^{m}$ is T-sequentially connected, then the information spread process of nodes is given by the spanning tree of the sequence of graphs $\left(\mathrm{G}_{i}\right)_{i=1}^{m}$. At the same time, if a sequence of graphs $\left(G_{i}\right)_{i=1}^{m}$ is sequentially connected, then it is also jointly connected, but the converse is not true.

\subsection{Model description}

Let $\mathrm{V}^{x}=\mathrm{V}^{y}=\{1,2, \ldots, N\}$ be the sets of nodes. In this paper, we study two interconnected bidirection MNNs system and consider each MNNs consisting of $N$ NNs. Each of the NNs corresponds to a node, and the state equations of the $i$ th and the $j$ th $\mathrm{NN}$ are given by

$$
\begin{aligned}
& \frac{d x_{i s}(t)}{d t}=-c_{s} x_{i s}(t)+\sum_{i=1}^{n} a_{s i} f_{i}\left(y_{j i}(t)\right)+\sum_{i=1}^{n} b_{s i} f_{i}^{\tau}\left(y_{j i}\left(t-\tau_{1}\right)\right)+I_{s}(t)+u_{i s}(t), \\
& \frac{d y_{j s}(t)}{d t}=-\mathfrak{c}_{s} y_{j s}(t)+\sum_{j=1}^{n} \mathfrak{a}_{s j} g_{j}\left(x_{i j}(t)\right)+\sum_{j=1}^{n} \mathfrak{b}_{s j} g_{j}^{\tau}\left(x_{i j}\left(t-\tau_{2}\right)\right)+\mathfrak{I}_{s}(t)+v_{j s}(t),
\end{aligned}
$$

or in compact forms

$$
\begin{aligned}
& \frac{d x_{i}(t)}{d t}=-C x_{i}(t)+A f\left(y_{j}(t)\right)+B f^{\tau}\left(y_{j}\left(t-\tau_{1}\right)\right)+I(t)+u_{i}(t), \\
& \frac{d y_{j}(t)}{d t}=-\mathfrak{C} y_{j}(t)+\mathfrak{A} g\left(x_{i}(t)\right)+\mathfrak{B} g^{\tau}\left(x_{i}\left(t-\tau_{2}\right)\right)+\mathfrak{I}(t)+v_{j}(t),
\end{aligned}
$$

where $i \in \mathrm{V}^{x}, j \in \mathrm{V}^{y}, t \geq t_{0}, x_{i}(t)=\left(x_{i 1}(t), x_{i 2}(t), \ldots, x_{i n}(t)\right) \in R^{n}$ and $y_{j}(t)=\left(y_{j 1}(t), y_{j 2}(t), \ldots\right.$, $\left.y_{j n}(t)\right) \in R^{n}$ are the state vectors of the $i$ th NN and $j$ th NN of the two MNNs, respectively. $C=\operatorname{diag}\left\{c_{1}, c_{2}, \ldots, c_{n}\right\}, c_{i}>0$ and $\mathfrak{C}=\operatorname{diag}\left\{\mathfrak{c}_{1}, \mathfrak{c}_{2}, \ldots, \mathfrak{c}_{n}\right\}, \mathfrak{c}_{i}>0$ are self-inhibitions of the two neurons. $I(t) \in R^{n}, \Im(t) \in R^{n}$ represent the input or bias, $u_{i}, v_{i}$ are control inputs of two neurons, respectively. $\tau_{1}>0, \tau_{2}>0$ are the transmission delays and let $\tau=\max \left\{\tau_{1}, \tau_{2}\right\}$. $f\left(y_{j}(t)\right)=\left(f_{1}\left(y_{j 1}(t)\right), f_{2}\left(y_{j 2}(t)\right), \ldots, f_{n}\left(y_{j n}(t)\right)\right)^{T}, g\left(x_{i}(t)\right)=\left(g_{1}\left(x_{i 1}(t)\right), g_{2}\left(x_{i 2}(t)\right), \ldots, g_{n}\left(x_{i n}(t)\right)\right)^{T}$ and $f^{\tau}\left(y_{j}\left(t-\tau_{1}\right)\right)=\left(f_{1}^{\tau}\left(y_{j 1}\left(t-\tau_{1}\right)\right), f_{2}^{\tau}\left(y_{j 2}\left(t-\tau_{1}\right)\right), \ldots, f_{n}^{\tau}\left(y_{j n}\left(t-\tau_{1}\right)\right)\right)^{T}, g^{\tau}\left(x_{i}\left(t-\tau_{2}\right)\right)=\left(g_{1}^{\tau} x_{i 1}(t-\right.$ $\left.\left.\tau_{2}\right), g_{2}^{\tau} x_{i 2}\left(t-\tau_{2}\right), \ldots, g_{n}^{\tau} x_{i n}\left(t-\tau_{2}\right)\right)^{T}$ are activation functions, and $A=\left[a_{i j}\right]_{n \times n}, \mathfrak{A}=\left[\mathfrak{a}_{i j}\right]_{n \times n}$, $B=\left[b_{i j}\right]_{n \times n}, \mathfrak{B}=\left[\mathfrak{b}_{i j}\right]_{n \times n}$ are the connection weight matrices and delay connection weight matrices of the two NNs.

Given an impulsive instant sequence $\left\{t_{1}, t_{2}, t_{3}, \ldots\right\}$ satisfying $0<t_{k}<t_{k+1}\left(k \in Z^{+}\right)$and $t_{k} \rightarrow \infty$ when $k \rightarrow \infty$. For the distributed impulsive controllers of the $i$ th and $j$ th nodes, 
we assign

$$
\begin{aligned}
& u_{i}(t)=\sum_{k=1}^{+\infty}\left(x_{i}(t)-\sum_{j=1}^{N} d_{i j}(t) \Gamma(t) x_{j}(t)\right) \delta\left(t-t_{k}\right), \\
& v_{j}(t)=\sum_{k=1}^{+\infty}\left(y_{j}(t)-\sum_{j=1}^{N} \mathfrak{d}_{j i}(t) \Lambda(t) y_{i}(t)\right) \delta\left(t-t_{k}\right),
\end{aligned}
$$

$\delta(\cdot)$ is the Dirac impulsive function; $\Gamma\left(t_{k}\right)=\operatorname{diag}\left\{\gamma_{1}\left(t_{k}\right), \gamma_{2}\left(t_{k}\right), \ldots, \gamma_{n}\left(t_{k}\right)\right\}$ and $\Lambda\left(t_{k}\right)=$ $\operatorname{diag}\left\{\lambda_{1}\left(t_{k}\right), \lambda_{2}\left(t_{k}\right), \ldots, \lambda_{n}\left(t_{k}\right)\right\}$ represent the coupling gains between two nodes with $\gamma_{i}\left(t_{k}\right)>$ 0 and $\lambda_{i}\left(t_{k}\right)>0 . D\left(t_{k}\right)=\left(d_{i j}\left(t_{k}\right)\right)_{N \times N}$ and $\mathfrak{D}\left(t_{k}\right)=\left(\mathfrak{d}_{j i}\left(t_{k}\right)\right)_{N \times N}$ represent the impulsive coupling matrices with

$$
\begin{aligned}
& \begin{cases}d_{i j}\left(t_{k}\right) \geq 0, & i \neq j, \\
\sum_{j=1}^{N} d_{i j}\left(t_{k}\right)=1, & \forall i \in \mathrm{V}^{x},\end{cases} \\
& \begin{cases}\mathfrak{d}_{i j}\left(t_{k}\right) \geq 0, & i \neq j, \\
\sum_{j=1}^{N} \mathfrak{d}_{i j}\left(t_{k}\right)=1, & \forall i \in \mathrm{V}^{y} .\end{cases}
\end{aligned}
$$

In algebraic graph theory, $\mathrm{G}\left(D\left(t_{k}\right)\right)$ and $\mathrm{G}\left(\mathfrak{D}\left(t_{k}\right)\right)$ are the adjacent matrices of the directed weight graphs $\mathrm{G}_{k}^{x}$ and $\mathrm{G}_{k}^{y}$.

Under impulsive controllers (2.5) and (2.6) with the conditions (2.7) and (2.8), then controlled systems (2.1) and (2.2) can be described in the following forms:

$$
\begin{aligned}
& \left\{\begin{array}{l}
\frac{d x_{i}(t)}{d t}=-C x_{i}(t)+A f\left(y_{j}(t)\right)+B f^{\tau}\left(y_{j}\left(t-\tau_{1}\right)\right)+I(t), \quad t \neq t_{k}, \\
x_{i}\left(t_{k}^{+}\right)=\sum_{j=1}^{N} d_{i j}\left(t_{k}\right) \Gamma\left(t_{k}\right) x_{j}\left(t_{k}\right),
\end{array}\right. \\
& \left\{\begin{array}{l}
\frac{d y_{j}(t)}{d t}=-\mathfrak{C}_{j}(t)+\mathfrak{A} g\left(x_{i}(t)\right)+\mathfrak{B} g^{\tau}\left(x_{i}\left(t-\tau_{2}\right)\right)+\mathfrak{I}(t), \quad t \neq t_{k}, \\
y_{j}\left(t_{k}^{+}\right)=\sum_{i=1}^{N} \mathfrak{d}_{j i}\left(t_{k}\right) \Lambda\left(t_{k}\right) y_{i}\left(t_{k}\right),
\end{array}\right.
\end{aligned}
$$

where $x_{i}\left(t_{k}^{-}\right)=x_{i}\left(t_{k}\right), y_{j}\left(t_{k}^{-}\right)=y_{j}\left(t_{k}\right)$.

We use $D\left(t_{k}\right)$ and $\mathfrak{D}\left(t_{k}\right)$ to describe the coupling topology of NNs (2.9) and (2.10) at impulsive time $t_{k}$. The graphs $\mathrm{G}_{k}^{x}$ and $\mathrm{G}_{k}^{y}$ correspond to matrices $D\left(t_{k}\right)$ and $\mathfrak{D}\left(t_{k}\right)$, respectively. Coupling matrices $D\left(t_{k}\right)$ and $\mathfrak{D}\left(t_{k}\right)$ can switch at impulsive time $t_{k}$ with period. It should be noted that $\mathrm{G}_{k}^{x}$ and $\mathrm{G}_{k}^{y}$ may contain selfloops, which means the conditions $d_{i i}\left(t_{k}\right)>0$ and $\mathfrak{d}_{i i}\left(t_{k}\right)>0$ exist.

Remark 2.2 Through (2.7) and (2.8) we can see that the couplings of the two MNNs only occur at the impulsive time $t_{k}$, and each node of the two MNNs is independent at other times. In addition, there is an interaction between the corresponding nodes of the two MNNs, so that there is also an interaction between the two MNNs. Then how to synchronize the two networks through certain conditions will be a problem we will discuss later.

Remark 2.3 Compared with the controllers used in articles [24-26], the controllers in these three articles are all used in single-layer network, but the system in this paper is a 
bidirection MNNs system, for which one needs to consider the coupling between nodes at the impulsive time, so this type of controllers may not be suitable. The controllers used in this paper can be regarded as a generalization of this kind of controller in MNNs.

Note that when each NNs in MNNs (2.9) and (2.10) satisfy

$$
\begin{aligned}
& \lim _{t \rightarrow \infty}\left\|x_{i}(t)-x_{j}(t)\right\|=0, \quad i, j \in \mathrm{V}^{x}, \\
& \lim _{t \rightarrow \infty}\left\|y_{i}(t)-y_{j}(t)\right\|=0, \quad i, j \in \mathrm{V}^{y},
\end{aligned}
$$

then MNNs (2.9) and (2.10) are globally synchronized.

Let $\gamma_{\max }=\max _{k \geq 1, i \in N}\left\{\gamma_{i}\left(t_{k}\right)\right\}, \lambda_{\max }=\max _{k \geq 1, i \in N}\left\{\lambda_{i}\left(t_{k}\right)\right\}$ and $\gamma=-\ln \left(\gamma_{\max }\right), \lambda=-\ln \left(\lambda_{\max }\right)$ are called coupling strength of $\mathrm{G}_{k}^{x}$ and $\mathrm{G}_{k}^{y}$.

For the continuous activation functions $f_{i}(x), g_{i}(x)$ and $f_{i}^{\tau}(x), g_{i}^{\tau}(x)$, Assumption 1 is made.

Assumption 1 There exist $p_{i}>0, p_{i}^{\tau}>0$ and $l_{j}>0, l_{j}^{\tau}>0$ such that

$$
\begin{aligned}
& \left|f_{i}(x)-f_{i}(y)\right| \leq p_{i}|x-y|, \\
& \left|f_{i}^{\tau}(x)-f_{i}^{\tau}(y)\right| \leq p_{i}^{\tau}|x-y|, \\
& \left|g_{j}(x)-g_{j}(y)\right| \leq l_{j}|x-y|, \\
& \left|g_{j}^{\tau}(x)-g_{j}^{\tau}(y)\right| \leq l_{j}^{\tau}|x-y|,
\end{aligned}
$$

for any $x, y \in R$ and $i=1,2, \ldots, n, j=1,2, \ldots, n$. Denote $P=\operatorname{diag}\left\{p_{1}, p_{2}, \ldots, p_{n}\right\}, P^{\tau}=$ $\operatorname{diag}\left\{p_{1}^{\tau}, p_{2}^{\tau}, \ldots, p_{n}^{\tau}\right\}$ and $L=\operatorname{diag}\left\{l_{1}, l_{2}, \ldots, l_{n}\right\}, L^{\tau}=\operatorname{diag}\left\{l_{1}^{\tau}, l_{2}^{\tau}, \ldots, l_{n}^{\tau}\right\}$.

\subsection{Definitions and properties}

In this paper, we give some concepts of the convex set [19]. Given a set $M \subseteq R^{n}$, then the convex hull of $M$ is defined as

$$
\overline{\mathrm{co}}(M)=\left\{\sum_{i=1}^{n} a_{i} x_{i}: \sum_{i=1}^{n} a_{i}=1, a_{i} \geq 0, x_{i} \in M, n \geq 1\right\} .
$$

We define the diameter of $M$ as

$$
\operatorname{diam}(M)=\sup _{x, y \in M}\|x-y\|
$$

Given two sets $M_{1}, M_{2} \subseteq R^{n}$, define

$$
M_{1}+M_{2}=\left\{x+y: x \in M_{1}, y \in M_{2}\right\} .
$$

It is obvious that $\operatorname{diam}\left(M_{1}+M_{2}\right) \leq \operatorname{diam}\left(M_{1}\right)+\operatorname{diam}\left(M_{2}\right)$.

Let $e_{i j}(t)=x_{i}(t)-x_{j}(t), i, j \in \mathrm{V}^{x}, \mathfrak{e}_{i j}(t)=y_{i}(t)-y_{j}(t), i, j \in \mathrm{V}^{y}$, The state error between NNs of (2.9) and (2.10) are defined as $\left\|e_{i j}(t)\right\|$ and $\left\|\mathfrak{e}_{i j}(t)\right\|,\|\cdot\|$ represent the 1-norm. Besides, let $\left\|\tilde{e}_{i j}(t)\right\|=\sup _{\theta_{1} \in[-\tau, 0]}\left\|e_{i j}\left(t+\theta_{1}\right)\right\|,\left\|\tilde{\mathfrak{e}}_{i j}(t)\right\|=\sup _{\theta_{2} \in[-\tau, 0]}\left\|\mathfrak{e}_{i j}\left(t+\theta_{2}\right)\right\|$ and $h_{k}=t_{k+1}-t_{k}, h_{\text {inf }}=$ $\inf _{k \in z^{+}}\left\{h_{k}\right\}, h_{\text {sup }}=\sup _{k \in z^{+}}\left\{h_{k}\right\}, 0 \leq \tau \leq h_{\text {inf }}$. 
Lemma 2.1 For any $i, j \in\{1,2, \ldots, N\}$ and $t \in\left(t_{k}, t_{k+1}\right], k \in Z^{+}$, we have

$$
\begin{aligned}
\left\|e_{i j}(t)\right\| & \leq\left\|\tilde{e}_{i j}\left(t_{k}^{+}\right)\right\| \exp \left\{-r\left(t-t_{k}\right)\right\}, \\
\left\|\mathfrak{e}_{i j}(t)\right\| & \leq\left\|\tilde{e}_{i j}\left(t_{k}^{+}\right)\right\| \exp \left\{-r\left(t-t_{k}\right)\right\},
\end{aligned}
$$

where $r \neq 0$ and satisfies

$$
\begin{aligned}
& r-c_{\text {min }}+\|A P\|+\left\|B P^{\tau}\right\| \exp \{r \tau\} \leq 0, \\
& r-\mathfrak{c}_{\text {min }}+\|\mathfrak{A} L\|+\left\|\mathfrak{B} L^{\tau}\right\| \exp \{r \tau\} \leq 0,
\end{aligned}
$$

in which $c_{\min }=\min _{1 \leq i \leq n}\left\{c_{i}\right\}, \mathfrak{c}_{\min }=\min _{1 \leq i \leq n}\left\{\mathfrak{c}_{i}\right\}$.

Proof Let

$$
\begin{aligned}
& \mathbb{V}(t)=\left\|e_{i j}(t)\right\| \exp \left\{r\left(t-t_{k}\right)\right\}, \\
& \mathfrak{V}(t)=\left\|\mathfrak{e}_{i j}(t)\right\| \exp \left\{r\left(t-t_{k}\right)\right\},
\end{aligned}
$$

and $\mathbb{W}(t)=\max \{\mathbb{V}(t), \mathfrak{V}(t)\}$.

According to (2.9) and (2.10), when $t \in\left(t_{k}, t_{k+1}\right)$, we have

$$
\begin{aligned}
\frac{d e_{i j}(t)}{d t}= & \frac{d x_{i}(t)}{d t}-\frac{d x_{j}(t)}{d t} \\
= & -C\left(x_{i}(t)-x_{j}(t)\right)+A\left[f\left(y_{j}(t)\right)-f\left(y_{i}(t)\right)\right] \\
& +B\left[f^{\tau}\left(y_{j}\left(t-\tau_{1}\right)\right)-f^{\tau}\left(y_{i}\left(t-\tau_{1}\right)\right)\right], \\
\frac{d \mathfrak{e}_{i j}(t)}{d t}= & \frac{d y_{i}(t)}{d t}-\frac{d y_{j}(t)}{d t} \\
= & -\mathfrak{C}\left(y_{i}(t)-y_{j}(t)\right)+\mathfrak{A}\left[g\left(x_{j}(t)\right)-g\left(x_{i}(t)\right)\right] \\
& +\mathfrak{B}\left[g^{\tau}\left(x_{j}\left(t-\tau_{2}\right)\right)-g^{\tau}\left(x_{i}\left(t-\tau_{2}\right)\right)\right] .
\end{aligned}
$$

Then

$$
\begin{aligned}
\frac{d \mathbb{V}(t)}{d t}= & \operatorname{sign}\left(e_{i j}(t)\right)^{T} \frac{d e_{i j}}{d t} \exp \left\{r\left(t-t_{k}\right)\right\}+r \mathbb{V}(t) \\
= & -\operatorname{sign}\left(e_{i j}(t)\right)^{T} C\left(x_{i}(t)-x_{j}(t)\right) \exp \left\{r\left(t-t_{k}\right)\right\} \\
& +r \mathbb{V}(t)+\operatorname{sign}\left(e_{i j}(t)\right)^{T} A\left[f\left(y_{j}(t)\right)-f\left(y_{i}(t)\right)\right] \exp \left\{r\left(t-t_{k}\right)\right\} \\
& +\operatorname{sign}\left(e_{i j}(t)\right)^{T} B\left[f^{\tau}\left(y_{j}\left(t-\tau_{1}\right)\right)-f^{\tau}\left(y_{i}\left(t-\tau_{1}\right)\right)\right] \exp \left\{r\left(t-t_{k}\right)\right\} \\
\leq & \left(r-c_{\min }\right) \mathbb{V}(t)+\|A P\|\left\|y_{i}(t)-y_{j}(t)\right\| \exp \left\{r\left(t-t_{k}\right)\right\} \\
& +\left\|B P^{\tau}\right\|\left\|y_{i}\left(t-\tau_{1}\right)-y_{j}\left(t-\tau_{1}\right)\right\| \exp \left\{r\left(t-t_{k}\right)\right\} \\
\leq & \left(r-c_{\min }\right) \mathbb{V}(t)+\|A P\| \mathfrak{V}(t)+\left\|B P^{\tau}\right\| \mathfrak{V}\left(t-\tau_{1}\right) \exp \{r \tau\} \\
\leq & \left(r-c_{\min }+\|A P\|\right) \mathbb{W}(t)+\left\|B P^{\tau}\right\| \mathbb{W}\left(t-\tau_{1}\right) \exp \{r \tau\} .
\end{aligned}
$$


Let

$$
\tilde{\mathbb{W}}(t)=\sup _{\theta \in[-\tau, 0]} \mathbb{W}(t+\theta) .
$$

Then one has

$$
\tilde{\mathbb{W}}(t) \geq \mathbb{W}(t) \geq 0
$$

From (2.13) and (2.15) we have

$$
\begin{aligned}
\frac{d \mathbb{V}(t)}{d t} & =\operatorname{sign}\left(e_{i j}(t)\right)^{T} \frac{d e_{i j}}{d t} \exp \left\{r\left(t-t_{k}\right)\right\}+r \mathbb{V}(t) \\
& \leq\left(r-c_{\min }+\|A P\|\right) \mathbb{W}(t)+\left\|B P^{\tau}\right\| \mathbb{W}\left(t-\tau_{1}\right) \exp \{r \tau\} \\
& \leq\left(r-c_{\min }+\|A P\|+\left\|B P^{\tau}\right\| \exp \{r \tau\}\right) \tilde{\mathbb{W}}(t) \\
& \leq 0 .
\end{aligned}
$$

Let $\tilde{\mathbb{V}}(t)=\sup _{\theta_{1} \in[-\tau, 0]} \mathbb{V}\left(t+\theta_{1}\right)$, from (2.16) and using the Lyapunov-Razumikhin method, we can get

$$
\frac{d \tilde{\mathbb{V}}(t)}{d t} \leq 0, \quad \forall t \in\left(t_{k}, t_{k+1}\right)
$$

then we can get

$$
\mathbb{V}(t) \leq \tilde{\mathbb{V}}(t) \leq \tilde{\mathbb{V}}\left(t_{k}^{+}\right)=\left\|\tilde{e}_{i j}\left(t_{k}^{+}\right)\right\|
$$

hence

$$
\left\|\tilde{e}_{i j}(t)\right\| \leq\left\|\tilde{e}_{i j}\left(t^{+}\right)\right\| \exp \left\{-r\left(t-t_{k}\right)\right\}
$$

Similarly we can get

$$
\begin{aligned}
\frac{d \mathfrak{V}(t)}{d t} & =\operatorname{sign}\left(\mathfrak{e}_{i j}(t)\right)^{T} \frac{d \mathfrak{e}_{i j}}{d t} \exp \left\{r\left(t-t_{k}\right)\right\}+r \mathfrak{V}(t) \\
& \leq\left(r-\mathfrak{c}_{\text {min }}+\|\mathfrak{A} L\|+\left\|\mathfrak{B} L^{\tau}\right\| \exp \{r \tau\}\right) \tilde{\mathbb{W}}(t) \\
& \leq 0
\end{aligned}
$$

and

$$
\left\|\tilde{\mathfrak{e}}_{i j}(t)\right\| \leq\left\|\tilde{\mathfrak{e}}_{i j}\left(t_{k}^{+}\right)\right\| \exp \left\{-r\left(t-t_{k}\right)\right\}
$$

which mean (2.11) and (2.12) are true.

Remark 2.4 The result of the Lemma 2.1 is important in the subsequent proof, it establishes the relationship of $\left\|e_{i j}(t)\right\|$ and $\left\|\mathfrak{e}_{i j}(t)\right\|$ in two adjacent impulsive interval, so we can go through the iteration to derive them up to time $t=t_{1}$. 


\section{Main results}

\subsection{Globally synchronization}

Theorem 3.1 Assume that Assumption 1 holds and the model satisfies the following conditions:

(1) There exist two integers $T \geq 1$ and $\mathfrak{T} \geq 1$, such that each sequence of graphs $\left\{\mathrm{G}_{j}^{x}\right\}_{j=m T+1}^{(m+1) T}$ and $\left\{\mathrm{G}_{j}^{y}\right\}_{j=m \mathfrak{T}+1}^{(m+1) \mathfrak{T}}$ are sequentially connected for $\forall m \in N^{+}$. Furthermore, for any $i \in \mathrm{V}_{k+1}^{x}, j \in \mathrm{V}_{k+1}^{y}$, there exist $\alpha_{1} \in(0,1)$ and $\alpha_{2} \in(0,1)$, satisfying

$$
\begin{aligned}
& \sum_{j \in \mathrm{V}_{k}^{x}} d_{i j}\left(t_{k}\right) \geq \alpha_{1}, \\
& \sum_{i \in \cup_{k}^{y}} \mathfrak{d}_{j i}\left(t_{k}\right) \geq \alpha_{2} .
\end{aligned}
$$

(2) $r \neq 0$ satisfies the inequalities (2.13), (2.14) and

$$
\begin{aligned}
& \rho^{T} \exp \left\{-r q_{m}-T \gamma\right\}\left(1-\left(\alpha_{1}\right)^{T}\right)<1, \\
& \rho^{\mathfrak{T}} \exp \left\{-r \mathfrak{q}_{m}-\mathfrak{T} \lambda\right\}\left(1-\left(\alpha_{2}\right)^{\mathfrak{T}}\right)<1,
\end{aligned}
$$

where $q_{m}=t_{(m+1) T+1}-t_{m T+1}, \mathfrak{q}_{m}=t_{(m+1) \mathfrak{T}+1}-t_{m \mathfrak{T}+1}$ and $\rho=\max \{1, \exp \{r \tau\}\} . \gamma$ and $\lambda$ are the coupling strength of $G^{x}$ and $G^{y}$, respectively, then MNNs (2.9) and (2.10) are globally synchronized.

Proof By the condition (1), we can see that the graph sequences $\left\{\mathrm{G}_{j}^{x}\right\}_{j=m T+1}^{(m+1) T}$ and $\left\{\mathrm{G}_{j}^{y}\right\}_{j=m \mathfrak{T}+1}^{(m+1) \mathfrak{T}}$ are sequentially connected.

First, consider the condition of $m=0$, namely, concentrating on the moments of $t=t_{k}$ with $k=1,2, \ldots, T$ or $k=1,2, \ldots, \mathfrak{T}$. Since $\left\{G_{k}^{x}\right\}_{k=1}^{T}$ and $\left\{G_{k}^{y}\right\}_{k=1}^{\mathfrak{T}}$ are sequentially connected, we may suppose that

$$
\begin{aligned}
& \mathrm{V}_{k+1}^{x} \subseteq \mathrm{V}_{k}^{x} \cup \mathrm{N}\left(\mathrm{G}_{k}^{x}, \mathrm{~V}_{k}^{x}\right), \\
& \mathrm{V}_{k+1}^{y} \subseteq \mathrm{V}_{k}^{y} \cup \mathrm{N}\left(\mathrm{G}_{k}^{y}, \mathrm{~V}_{k}^{y}\right),
\end{aligned}
$$

where $\mathrm{V}_{1}^{x}, \mathrm{~V}_{1}^{y}$ are singletons and $\mathrm{V}_{T+1}^{x}=\mathrm{V}^{x}, \mathrm{~V}_{\mathfrak{T}+1}^{y}=\mathrm{V}^{y}$. For any $i \in \mathrm{V}_{k+1}^{x}$ and $j \in \mathrm{V}_{k+1}^{y}$, from (2.9) and (2.10) we have

$$
\begin{aligned}
x_{i}\left(t_{k}^{+}\right) & =\sum_{j=1}^{N} d_{i j}\left(t_{k}\right) \Gamma\left(t_{k}\right) x_{j}\left(t_{k}\right) \\
& =\sum_{j \in \mathrm{V}_{k}^{x}} d_{i j}\left(t_{k}\right) \Gamma\left(t_{k}\right) x_{j}\left(t_{k}\right)+\sum_{j \in \mathrm{V}^{x} \backslash \mathrm{V}_{k}^{x}} d_{i j}\left(t_{k}\right) \Gamma\left(t_{k}\right) x_{j}\left(t_{k}\right), \\
y_{j}\left(t_{k}^{+}\right) & =\sum_{i=1}^{N} \mathfrak{d}_{j i}\left(t_{k}\right) \Lambda\left(t_{k}\right) y_{i}\left(t_{k}\right) \\
& =\sum_{i \in \mathrm{V}_{k}^{y}} \mathfrak{d}_{j i}\left(t_{k}\right) \Lambda\left(t_{k}\right) y_{i}\left(t_{k}\right)+\sum_{i \in \mathrm{V}^{y} \backslash \mathrm{V}_{k}^{y}} \mathfrak{d}_{j i}\left(t_{k}\right) \Lambda\left(t_{k}\right) y_{i}\left(t_{k}\right) .
\end{aligned}
$$


Let $\zeta_{i}\left(t_{k}\right)=\sum_{j \in \mathrm{V}_{k}^{x}} d_{i j}\left(t_{k}\right), \eta_{j}\left(t_{k}\right)=\sum_{i \in \mathrm{V}_{k}^{y}} \mathfrak{d}_{j i}\left(t_{k}\right)$ and $\hat{d}_{i j}\left(t_{k}\right)=\frac{1}{\zeta_{i}\left(t_{k}\right)} d_{i j}\left(t_{k}\right), \hat{\mathfrak{d}}_{j i}\left(t_{k}\right)=\frac{1}{\eta_{j}\left(t_{k}\right)} \mathfrak{j}_{j i}\left(t_{k}\right)$, then we have $\sum_{j \in \mathrm{V}_{k}^{x}} \hat{d}_{i j}\left(t_{k}\right)=1, \sum_{i \in \mathrm{V}_{k}^{y}} \hat{\mathfrak{d}}_{j i}\left(t_{k}\right)=1$ and

$$
\begin{aligned}
& \sum_{j \in \mathrm{V}_{k}^{x}} d_{i j}\left(t_{k}\right) \Gamma\left(t_{k}\right) x_{j}\left(t_{k}\right)=\zeta_{i}\left(t_{k}\right) \sum_{j \in \mathrm{V}_{k}^{x}} \hat{d}_{i j}\left(t_{k}\right) \Gamma\left(t_{k}\right) x_{j}\left(t_{k}\right), \\
& \sum_{i \in \mathrm{V}_{k}^{y}} \mathfrak{d}_{j i}\left(t_{k}\right) \Lambda\left(t_{k}\right) y_{i}\left(t_{k}\right)=\eta_{j}\left(t_{k}\right) \sum_{i \in \mathrm{V}_{k}^{y}} \hat{\mathfrak{d}}_{j i}\left(t_{k}\right) \Lambda\left(t_{k}\right) y_{i}\left(t_{k}\right) .
\end{aligned}
$$

Similarly, denote $\phi_{i}\left(t_{k}\right)=1-\zeta_{i}\left(t_{k}\right)>0, \psi_{j}\left(t_{k}\right)=1-\eta_{j}\left(t_{k}\right)>0$ and

$$
\begin{aligned}
& \check{d}_{i j}\left(t_{k}\right)= \begin{cases}\frac{d_{i j}\left(t_{k}\right)}{\phi\left(t_{k}\right)}, & j \in \mathrm{V}^{x} \backslash \mathrm{V}_{k}^{x}, \\
0, & j \in \mathrm{V}_{k}^{x},\end{cases} \\
& \check{\mathfrak{d}}_{j i}\left(t_{k}\right)= \begin{cases}\frac{\mathfrak{o}_{j i}\left(t_{k}\right)}{\psi\left(t_{k}\right)}, & i \in \mathrm{V}^{y} \backslash \mathrm{V}_{k}^{y}, \\
0, & i \in \mathrm{V}_{k}^{y} .\end{cases}
\end{aligned}
$$

Obviously

$$
\begin{aligned}
& \sum_{j \in \mathrm{V}^{x}} d_{i j}\left(t_{k}\right)=\sum_{j \in \mathrm{V}^{x} \backslash \mathrm{V}_{k}^{x}} d_{i j}\left(t_{k}\right)+\sum_{j \in \mathrm{V}_{k}^{x}} d_{i j}\left(t_{k}\right)=1, \\
& \sum_{i \in \mathrm{V}^{y}} \mathfrak{d}_{j i}\left(t_{k}\right)=\sum_{i \in \mathrm{V}^{y} \backslash \mathrm{V}_{k}^{y}} \mathfrak{d}_{j i}\left(t_{k}\right)+\sum_{i \in \mathrm{V}_{k}^{y}} \mathfrak{d}_{j i}\left(t_{k}\right)=1,
\end{aligned}
$$

then we have

$$
\begin{aligned}
\sum_{j \in \mathrm{V}^{x} \backslash \mathrm{V}_{k}^{x}} \check{d}_{i j}\left(t_{k}\right) & =\frac{\sum_{j \in \mathrm{V}^{x} \backslash \mathrm{V}_{k}^{x}} d_{i j}\left(t_{k}\right)}{\phi\left(t_{k}\right)} \\
& =\frac{\sum_{j \in \mathrm{V}^{x} \backslash \mathrm{V}_{k}^{x}} d_{i j}\left(t_{k}\right)}{1-\zeta_{i}\left(t_{k}\right)} \\
& =\frac{\sum_{j \in \mathrm{V}^{x} \backslash \mathrm{V}_{k}^{x}} d_{i j}\left(t_{k}\right)}{1-\sum_{j \in \mathrm{V}_{k}^{x}} d_{i j}\left(t_{k}\right)} \\
& =1, \\
\sum_{i \in \mathrm{V}^{y} \backslash \mathrm{V}_{k}^{y}} \check{\mathfrak{d}}_{j i}\left(t_{k}\right) & =\frac{\sum_{i \in \mathrm{V}^{y} \backslash \mathrm{V}_{k}^{y}}^{y} \mathfrak{d}_{j i}\left(t_{k}\right)}{\phi\left(t_{k}\right)} \\
& =\frac{\sum_{i \in \mathrm{V}^{y} \backslash \mathrm{V}_{k}^{y}} \mathfrak{d}_{j i}\left(t_{k}\right)}{1-\eta_{j}\left(t_{k}\right)} \\
& =\frac{\sum_{i \in \mathrm{V}^{y} \backslash \mathrm{V}_{k}^{y}} \mathfrak{d}_{j i}\left(t_{k}\right)}{1-\sum_{i \in \mathrm{V}_{k}^{y}} \mathfrak{d}_{j i}\left(t_{k}\right)} \\
& =1,
\end{aligned}
$$


and

$$
\begin{aligned}
\sum_{j \in \mathrm{V}^{x} \backslash \mathrm{V}_{k}^{x}} d_{i j}\left(t_{k}\right) \Gamma\left(t_{k}\right) x_{j}\left(t_{k}\right) & =\phi_{i}\left(t_{k}\right) \sum_{j \in \mathrm{V}^{x} \backslash \mathrm{V}_{k}^{x}} \check{d}_{i j}\left(t_{k}\right) \Gamma\left(t_{k}\right) x_{j}\left(t_{k}\right) \\
& =\phi_{i}\left(t_{k}\right) \sum_{j=1}^{N} \check{d}_{i j}\left(t_{k}\right) \Gamma\left(t_{k}\right) x_{j}\left(t_{k}\right), \\
\sum_{i \in \mathrm{V}^{y} \backslash \mathrm{V}_{k}^{y}} \mathfrak{d}_{j i}\left(t_{k}\right) \Lambda\left(t_{k}\right) y_{i}\left(t_{k}\right) & =\psi_{j}\left(t_{k}\right) \sum_{i \in \mathrm{V}^{y} \backslash \mathrm{V}_{k}^{y}} \check{\mathfrak{d}}_{j i}\left(t_{k}\right) \Lambda\left(t_{k}\right) y_{i}\left(t_{k}\right) \\
& =\psi_{j}\left(t_{k}\right) \sum_{i=1}^{N} \check{\mathfrak{d}}_{j i}\left(t_{k}\right) \Lambda\left(t_{k}\right) y_{i}\left(t_{k}\right) .
\end{aligned}
$$

From (3.1), (3.2), we can get $\zeta_{i}\left(t_{k}\right) \geq \alpha_{1}$ and $\eta_{j}\left(t_{k}\right) \geq \alpha_{2}$, combining (3.5), (3.7), (3.9) and (3.6), (3.8), (3.10) we have

$$
\begin{aligned}
x_{i}\left(t_{k}^{+}\right)= & \sum_{j=1}^{N} d_{i j}\left(t_{k}\right) \Gamma\left(t_{k}\right) x_{j}\left(t_{k}\right) \\
= & \alpha_{1} \sum_{j \in \mathrm{V}_{k}^{x}} \hat{d}_{i j}\left(t_{k}\right) \Gamma\left(t_{k}\right) x_{j}\left(t_{k}\right)+\left(\zeta_{i}\left(t_{k}\right)-\alpha_{1}\right) \sum_{j \in \mathrm{V}_{k}^{x}} \hat{d}_{i j}\left(t_{k}\right) \Gamma\left(t_{k}\right) x_{j}\left(t_{k}\right) \\
& +\left(1-\zeta_{i}\left(t_{k}\right)\right) \sum_{j=1}^{N} \check{d}_{i j}\left(t_{k}\right) \Gamma\left(t_{k}\right) x_{j}\left(t_{k}\right), \\
y_{j}\left(t_{k}^{+}\right)= & \sum_{i=1}^{N} \mathfrak{d}_{j i}\left(t_{k}\right) \Lambda\left(t_{k}\right) y_{i}\left(t_{k}\right) \\
= & \alpha_{2} \sum_{i \in \mathrm{V}_{k}^{y}} \hat{\mathfrak{d}}_{j i}\left(t_{k}\right) \Lambda\left(t_{k}\right) y_{i}\left(t_{k}\right)+\left(\eta_{j}\left(t_{k}\right)-\alpha_{2}\right) \sum_{i \in \mathrm{V}_{k}^{y}} \hat{\mathfrak{d}}_{j i}\left(t_{k}\right) \Lambda\left(t_{k}\right) y_{i}\left(t_{k}\right) \\
& +\left(1-\eta_{j}\left(t_{k}\right)\right) \sum_{i=1}^{N} \check{\mathfrak{d}}_{j i}\left(t_{k}\right) \Lambda\left(t_{k}\right) y_{i}\left(t_{k}\right) .
\end{aligned}
$$

Let

$$
\begin{aligned}
& \bar{d}_{i j}\left(t_{k}\right)= \begin{cases}\frac{\left[\zeta_{i}\left(t_{k}\right)-\alpha_{1}\right] \hat{d}_{i j}\left(t_{k}\right)}{1-\alpha_{1}}, & j \in \mathrm{V}_{k}^{x}, \\
\frac{\left.\left[1-\zeta_{i}\left(t_{k}\right)\right]\right]_{i j}\left(t_{k}\right)}{1-\alpha_{1}}, & j \in \mathrm{V}^{x} \backslash \mathrm{V}_{k}^{x},\end{cases} \\
& \overline{\mathfrak{d}}_{j i}\left(t_{k}\right)= \begin{cases}\frac{\left[\eta_{j}\left(t_{k}\right)-\alpha_{2}\right] \hat{\mathfrak{d}}_{j i}\left(t_{k}\right)}{1-\alpha_{2}}, & i \in \mathrm{V}_{k}^{y}, \\
\frac{\left[1-\eta_{j}\left(t_{k}\right)\right] \mathfrak{d}_{j i}\left(t_{k}\right)}{1-\alpha_{2}}, & i \in \mathrm{V}^{y} \backslash \mathrm{V}_{k}^{y},\end{cases}
\end{aligned}
$$

then we get

$$
\begin{aligned}
\sum_{j=1}^{N} \bar{d}_{i j}\left(t_{k}\right) & =\sum_{j \in \mathrm{V}_{k}^{x}} \bar{d}_{i j}\left(t_{k}\right)+\sum_{j \in \mathrm{V}^{x} \backslash \mathrm{V}_{k}^{x}} \bar{d}_{i j}\left(t_{k}\right) \\
& =\frac{\left[\zeta_{i}\left(t_{k}\right)-\alpha_{1}\right] \sum_{j \in \mathrm{V}_{k}^{x}} \hat{d}_{i j}\left(t_{k}\right)}{1-\alpha_{1}}+\frac{\left[1-\zeta_{i}\left(t_{k}\right)\right] \sum_{j \in \mathrm{V}^{x} \backslash \mathrm{V}_{k}^{x}} \check{d}_{i j}\left(t_{k}\right)}{1-\alpha_{1}}
\end{aligned}
$$




$$
\begin{aligned}
& =\frac{\left[\zeta_{i}\left(t_{k}\right)-\alpha_{1}\right]+\left[1-\zeta_{i}\left(t_{k}\right)\right]}{1-\alpha_{1}} \\
& =1 \\
\sum_{i=1}^{N} \overline{\mathfrak{d}}_{j i}\left(t_{k}\right) & =\sum_{i \in \mathrm{V}_{k}^{y}} \overline{\mathfrak{d}}_{j i}\left(t_{k}\right)+\sum_{i \in \mathrm{V} y \mathrm{~V}_{k}^{y}} \overline{\mathfrak{d}}_{j i}\left(t_{k}\right) \\
& =\frac{\left[\eta_{j}\left(t_{k}\right)-\alpha_{2}\right] \sum_{i \in \mathrm{V}_{k}^{y}} \hat{\mathfrak{d}}_{j i}\left(t_{k}\right)}{1-\alpha_{2}}+\frac{\left[1-\eta_{j}\left(t_{k}\right)\right] \sum_{y \in \mathrm{V}^{y} \backslash \mathrm{V}_{k}^{y}} \check{\mathfrak{d}}_{j i}\left(t_{k}\right)}{1-\alpha_{2}} \\
& =\frac{\left[\eta_{j}\left(t_{k}\right)-\alpha_{2}\right]+\left[1-\eta_{j}\left(t_{k}\right)\right]}{1-\alpha_{2}} \\
& =1,
\end{aligned}
$$

and we can write (3.5), (3.6) as follows:

$$
\begin{aligned}
x_{i}\left(t_{k}^{+}\right)= & \alpha_{1} \sum_{j \in \mathrm{V}_{k}^{x}} \hat{d}_{i j}\left(t_{k}\right) \Gamma\left(t_{k}\right) x_{j}\left(t_{k}\right)+\left(\zeta_{i}\left(t_{k}\right)-\alpha_{1}\right) \sum_{j \in \mathrm{V}_{k}^{x}} \hat{d}_{i j}\left(t_{k}\right) \Gamma\left(t_{k}\right) x_{j}\left(t_{k}\right) \\
& +\left(1-\zeta_{i}\left(t_{k}\right)\right) \sum_{j=1}^{N} \check{d}_{i j}\left(t_{k}\right) \Gamma\left(t_{k}\right) x_{j}\left(t_{k}\right) \\
= & \alpha_{1} \sum_{j \in \mathrm{V}_{k}^{x}} \hat{d}_{i j}\left(t_{k}\right) \Gamma\left(t_{k}\right) x_{j}\left(t_{k}\right)+\left(1-\alpha_{1}\right) \sum_{j=1}^{N} \bar{d}_{i j}\left(t_{k}\right) \Gamma\left(t_{k}\right) x_{j}\left(t_{k}\right), \\
y_{j}\left(t_{k}^{+}\right)= & \alpha_{2} \sum_{i \in \mathrm{V}_{k}^{y}} \hat{\mathfrak{d}}_{j i}\left(t_{k}\right) \Lambda\left(t_{k}\right) y_{i}\left(t_{k}\right)+\left(\eta_{j}\left(t_{k}\right)-\alpha_{2}\right) \sum_{i \in \mathrm{V}_{k}^{y}} \hat{\mathfrak{d}}_{j i}\left(t_{k}\right) \Lambda\left(t_{k}\right) y_{i}\left(t_{k}\right) \\
& +\left(1-\eta_{j}\left(t_{k}\right)\right) \sum_{i=1}^{N} \check{\mathfrak{d}}_{j i}\left(t_{k}\right) \Lambda\left(t_{k}\right) y_{i}\left(t_{k}\right) \\
= & \alpha_{2} \sum_{i \in \mathrm{V}_{k}^{y}} \hat{\mathfrak{d}}_{j i}\left(t_{k}\right) \Lambda\left(t_{k}\right) y_{i}\left(t_{k}\right)+\left(1-\alpha_{2}\right) \sum_{i=1}^{N} \overline{\mathfrak{d}}_{j i}\left(t_{k}\right) \Lambda\left(t_{k}\right) y_{i}\left(t_{k}\right) .
\end{aligned}
$$

Let

$$
\begin{aligned}
& \mathcal{S}_{k}(t)=\overline{\mathrm{co}}\left(\left(x_{i}(t)\right)_{i \in \mathrm{V}_{k}^{x}}\right), \\
& \mathcal{S}(t)=\overline{\mathrm{co}}\left(\left(x_{i}(t)\right)_{i \in \mathrm{V}^{x}}\right), \\
& \mathcal{S}_{k}^{+}\left(t_{k}^{+}\right)=\overline{\mathrm{co}}\left(\left(x_{i}\left(t_{k}^{+}\right)\right)_{i \in \mathrm{V}_{k+1}^{x}}\right)
\end{aligned}
$$

and

$$
\begin{aligned}
& \mathcal{M}_{k}(t)=\overline{\mathrm{co}}\left(\left(y_{j}(t)\right)_{j \in \mathrm{V}_{k}^{y}}\right), \\
& \mathcal{M}(t)=\overline{\mathrm{co}}\left(\left(y_{j}(t)\right)_{j \in \mathrm{V}^{y}}\right), \\
& \mathcal{M}_{k}^{+}\left(t_{k}^{+}\right)=\overline{\mathrm{co}}\left(\left(y_{j}\left(t_{k}^{+}\right)\right)_{j \in \mathrm{V}_{k+1}^{y}}\right) .
\end{aligned}
$$


Then we have

$$
\begin{aligned}
& x_{i}\left(t_{k}^{+}\right) \in \alpha_{1} \Gamma\left(t_{k}\right) \mathcal{S}\left(t_{k}\right)+\left(1-\alpha_{1}\right) \Gamma\left(t_{k}\right) \mathcal{S}\left(t_{k}\right), \\
& y_{j}\left(t_{k}^{+}\right) \in \alpha_{2} \Lambda\left(t_{k}\right) \mathcal{M}_{k}\left(t_{k}\right)+\left(1-\alpha_{2}\right) \Lambda\left(t_{k}\right) \mathcal{M}\left(t_{k}\right) .
\end{aligned}
$$

We can also get

$$
\begin{aligned}
& \mathcal{S}_{k}^{+}\left(t_{k}^{+}\right) \subseteq \alpha_{1} \Gamma\left(t_{k}\right) \mathcal{S}\left(t_{k}\right)+\left(1-\alpha_{1}\right) \Gamma\left(t_{k}\right) \mathcal{S}\left(t_{k}\right), \\
& \mathcal{M}_{k}^{+}\left(t_{k}^{+}\right) \subseteq \alpha_{2} \Lambda\left(t_{k}\right) \mathcal{M}_{k}\left(t_{k}\right)+\left(1-\alpha_{2}\right) \Lambda\left(t_{k}\right) \mathcal{M}\left(t_{k}\right) .
\end{aligned}
$$

Furthermore, let

$$
\begin{aligned}
& \left\{\begin{array}{l}
\Delta_{k}=\operatorname{diam}\left(\mathcal{S}_{k}\left(t_{k}\right)\right), \quad \Delta_{k}^{+}=\operatorname{diam}\left(\mathcal{S}_{k}^{+}\left(t_{k}^{+}\right)\right), \\
E_{k}=\operatorname{diam}\left(\mathcal{S}\left(t_{k}\right)\right), \quad E_{k}^{+}=\operatorname{diam}\left(\mathcal{S}\left(t_{k}^{+}\right)\right), \\
\tilde{\Delta}_{k}=\sup _{\theta_{1} \in\left[-\tau_{1}, 0\right]} \operatorname{diam}\left(\mathcal{S}_{k}\left(t_{k}+\theta_{1}\right)\right), \quad \tilde{\Delta}_{k}^{+}=\sup _{\theta_{1} \in\left[-\tau_{1}, 0\right]} \operatorname{diam}\left(\mathcal{S}_{k}^{+}\left(t_{k}^{+}+\theta_{1}\right)\right), \\
\tilde{E}_{k}=\sup _{\theta_{1} \in\left[-\tau_{1}, 0\right]} \operatorname{diam}\left(\mathcal{S}\left(t_{k}+\theta_{1}\right)\right), \quad \tilde{E}_{k}^{+}=\sup _{\theta_{1} \in\left[-\tau_{1}, 0\right]} \operatorname{diam}\left(\mathcal{S}\left(t_{k}^{+}+\theta_{1}\right)\right),
\end{array}\right. \\
& \left\{\begin{array}{l}
\nabla_{k}=\operatorname{diam}\left(\mathcal{M}_{k}\left(t_{k}\right)\right), \quad \nabla_{k}^{+}=\operatorname{diam}\left(\mathcal{M}_{k}^{+}\left(t_{k}^{+}\right)\right), \\
R_{k}=\operatorname{diam}\left(\mathcal{M}\left(t_{k}\right)\right), \quad R_{k}^{+}=\operatorname{diam}\left(\mathcal{M}\left(t_{k}^{+}\right)\right), \\
\tilde{\nabla}_{k}=\sup _{\theta_{2} \in\left[-\tau_{2}, 0\right]} \operatorname{diam}\left(\mathcal{M}_{k}\left(t_{k}+\theta_{2}\right)\right), \quad \tilde{\nabla}_{k}^{+}=\sup _{\theta_{2} \in\left[-\tau_{2}, 0\right]} \operatorname{diam}\left(\mathcal{M}{ }_{k}^{+}\left(t_{k}^{+}+\theta_{2}\right)\right), \\
\tilde{R}_{k}=\sup _{\theta_{2} \in\left[-\tau_{2}, 0\right]} \operatorname{diam}\left(\mathcal{M}\left(t_{k}+\theta_{2}\right)\right), \quad \tilde{R}_{k}^{+}=\sup _{\theta_{2} \in\left[-\tau_{2}, 0\right]} \operatorname{diam}\left(\mathcal{M}\left(t_{k}^{+}+\theta_{2}\right)\right) .
\end{array}\right.
\end{aligned}
$$

From (3.11) and (3.12) we can get

$$
\begin{aligned}
\Delta_{k}^{+} & \leq \gamma_{\max } \alpha_{1} \Delta_{k}+\gamma_{\max }\left(1-\alpha_{1}\right) E_{k}, \\
\nabla_{k}^{+} & \leq \lambda_{\max } \alpha_{2} \nabla_{k}+\lambda_{\max }\left(1-\alpha_{2}\right) R_{k} .
\end{aligned}
$$

According to Lemma 2.1 we have

$$
\begin{gathered}
\left\{\begin{array}{l}
\Delta_{k+1} \leq \tilde{\Delta}_{k+1} \leq \exp \left\{-r\left(t-t_{k}\right)\right\} \tilde{\Delta}_{k}^{+} \leq \rho \exp \left\{-r\left(t_{k+1}-t_{k}\right)\right\} \tilde{\Delta}_{k}^{+}, \\
E_{k+1} \leq \tilde{E}_{k+1} \leq \exp \left\{-r\left(t-t_{k}\right)\right\} \tilde{E}_{k}^{+} \leq \rho \exp \left\{-r\left(t_{k+1}-t_{k}\right)\right\} \tilde{E}_{k}^{+}
\end{array}\right. \\
\left\{\begin{array}{l}
\nabla_{k+1} \leq \tilde{\nabla}_{k+1} \leq \exp \left\{-r\left(t-t_{k}\right)\right\} \tilde{\nabla}_{k}^{+} \leq \rho \exp \left\{-r\left(t_{k+1}-t_{k}\right)\right\} \tilde{\nabla}_{k}^{+}, \\
R_{k+1} \leq \tilde{R}_{k+1} \leq \exp \left\{-r\left(t-t_{k}\right)\right\} \tilde{R}_{k}^{+} \leq \rho \exp \left\{-r\left(t_{k+1}-t_{k}\right)\right\} \tilde{R}_{k}^{+}
\end{array}\right.
\end{gathered}
$$

since $\max \left\{\tau_{1}, \tau_{2}\right\} \leq \tau \leq t_{k+1}-t_{k}$. In addition, according to (2.9), (2.10), we have

$$
\begin{aligned}
& \tilde{E}_{k}^{+} \leq \gamma_{\max } \tilde{E}_{k}, \\
& \tilde{R}_{k}^{+} \leq \lambda_{\max } \tilde{R}_{k} .
\end{aligned}
$$

Combining (3.13), (3.15), (3.17) and by iterations, one obtains

$$
\tilde{\Delta}_{k}^{+} \leq \gamma_{\max } \alpha_{1} \tilde{\Delta}_{k}+\gamma_{\max }\left(1-\alpha_{1}\right) \tilde{E}_{k}
$$




$$
\begin{aligned}
\leq & \rho \exp \left\{-r\left(t_{k}-t_{k-1}\right)\right\} \gamma_{\max } \times\left[\alpha_{1} \tilde{\Delta}_{k-1}^{+}+\gamma_{\max }\left(1-\alpha_{1}\right) \tilde{E}_{k-1}\right] \\
\leq & \rho^{2} \exp \left\{-r\left(t_{k}-t_{k-2}\right)\right\} \gamma_{\max }^{2} \times\left[\alpha_{1}^{2} \tilde{\Delta}_{k-2}^{+}+\gamma_{\max }\left(1-\alpha_{1}^{2}\right) \tilde{E}_{k-2}\right] \\
& \ldots \\
\leq & \rho^{\nu_{1}} \exp \left\{-r\left(t_{k}-t_{k-v_{1}}\right)\right\} \gamma_{\max }^{\nu_{1}} \times\left[\alpha_{1}^{\nu_{1}} \tilde{\Delta}_{k-\nu_{1}}^{+}+\gamma_{\max }\left(1-\alpha_{1}^{\nu_{1}}\right) \tilde{E}_{k-\nu_{1}}\right]
\end{aligned}
$$

for any $1 \leq v_{1} \leq k \leq T$.

Also combining (3.14), (3.16), (3.18) we can get

$$
\begin{aligned}
\tilde{\nabla}_{k}^{+} & \leq \lambda_{\max } \alpha_{2} \tilde{\nabla}_{k}+\lambda_{\max }\left(1-\alpha_{2}\right) \tilde{R}_{k} \\
& \leq \rho \exp \left\{-r\left(t_{k}-t_{k-1}\right)\right\} \lambda_{\max } \times\left[\alpha_{2} \tilde{\nabla}_{k-1}^{+}+\lambda_{\max }\left(1-\alpha_{2}\right) \tilde{R}_{k-1}\right] \\
& \leq \rho^{\nu_{2}} \exp \left\{-r\left(t_{k}-t_{k-v_{2}}\right)\right\} \lambda_{\max }^{\nu_{2}} \times\left[\alpha_{2}^{\nu_{2}} \tilde{\nabla}_{k-v_{2}}^{+}+\lambda_{\max }\left(1-\alpha_{2}^{\nu_{2}}\right) \tilde{R}_{k-v_{2}}\right]
\end{aligned}
$$

for any $1 \leq v_{2} \leq k \leq \mathfrak{T}$.

So, when $k=T, v_{1}=T-1$ and $k=\mathfrak{T}, v_{2}=\mathfrak{T}-1$, from (3.15) and (3.16), we can get

$$
\begin{aligned}
\tilde{\Delta}_{T+1} & \leq \exp \left\{-r\left(t_{T+1}-t_{T}\right)\right\} \tilde{\Delta}_{T}^{+} \\
& \leq \rho^{T} \exp \left\{-r q_{0}\right\} \gamma_{\max }^{T-1}\left[\alpha_{1}^{T-1} \tilde{\Delta}_{1}^{+}+\gamma_{\max }\left(1-\alpha_{1}^{T-1}\right) \tilde{E}_{1}\right], \\
\tilde{\nabla}_{\mathfrak{T}+1} & \leq \exp \left\{-r\left(t_{\mathfrak{T}+1}-t_{\mathfrak{T}}\right)\right\} \tilde{\nabla}_{\mathfrak{T}}^{+} \\
& \leq \rho^{\mathfrak{T}} \exp \left\{-r \mathfrak{q}_{0}\right\} \lambda_{\max }^{\mathfrak{T}-1}\left[\alpha_{2}^{\mathfrak{T}-1} \tilde{\nabla}_{1}^{+}+\lambda_{\max }\left(1-\alpha_{2}^{\mathfrak{T}-1}\right) \tilde{R}_{1}\right] .
\end{aligned}
$$

Since $\mathrm{V}_{1}^{x}$ and $\mathrm{V}_{1}^{y}$ are singletons, $\tilde{\Delta}_{1}=0, \tilde{\nabla}_{1}=0$.

Therefore

$$
\tilde{\Delta}_{1}^{+} \leq \gamma_{\max } \alpha_{1} \tilde{\Delta}_{1}+\gamma_{\max }\left(1-\alpha_{1}\right) \tilde{E}_{1} \leq \gamma_{\max }\left(1-\alpha_{1}\right) \tilde{E}_{1}
$$

and

$$
\tilde{\nabla}_{1}^{+} \leq \lambda_{\max } \alpha_{2} \tilde{\nabla}_{1}+\lambda_{\max }\left(1-\alpha_{2}\right) \tilde{R}_{1} \leq \lambda_{\max }\left(1-\alpha_{2}\right) \tilde{R}_{1} .
$$

Therefore, (3.19) and (3.21) imply

$$
\begin{aligned}
\tilde{\Delta}_{T+1} & \leq \rho^{T} \exp \left\{-r q_{0}\right\} \gamma_{\max }^{T-1}\left[\alpha_{1}^{T-1} \gamma_{\max }\left(1-\alpha_{1}\right) \tilde{E}_{1}+\gamma_{\max }\left(1-\alpha_{1}^{T-1}\right) \tilde{E}_{1}\right] \\
& \leq \rho^{T} \exp \left\{-r q_{0}\right\} \gamma_{\max }^{T}\left(1-\alpha_{1}^{T}\right) \tilde{E}_{1} \\
& \leq \rho^{T} \exp \left\{-r q_{0}-T \gamma\right\}\left(1-\alpha_{1}^{T}\right) \tilde{E}_{1},
\end{aligned}
$$

from (3.20) and (3.22) we can get

$$
\begin{aligned}
\tilde{\nabla}_{\mathfrak{T}+1} & \leq \rho^{\mathfrak{T}} \exp \left\{-r q_{0}\right\} \lambda_{\max }^{\mathfrak{T}-1}\left[\alpha_{2}^{\mathfrak{T}-1} \lambda_{\max }\left(1-\alpha_{2}\right) \tilde{R}_{1}+\lambda_{\max }\left(1-\alpha_{2}^{\mathfrak{T}-1}\right) \tilde{R}_{1}\right] \\
& \leq \rho^{\mathfrak{T}} \exp \left\{-r \mathfrak{q}_{0}\right\} \lambda_{\max }^{\mathfrak{T}}\left(1-\alpha_{2}^{\mathfrak{T}}\right) \tilde{R}_{1} \\
& \leq \rho^{\mathfrak{T}} \exp \left\{-r \mathfrak{q}_{0}-\mathfrak{T} \lambda\right\}\left(1-\alpha_{2}^{\mathfrak{T}}\right) \tilde{R}_{1} .
\end{aligned}
$$


It follows from (3.23) and (3.24) that

$$
\begin{aligned}
& \tilde{\Delta}_{T+1} \leq \epsilon_{1} \tilde{E}_{1}, \\
& \tilde{\nabla}_{\mathfrak{T}+1} \leq \epsilon_{2} \tilde{R}_{1},
\end{aligned}
$$

where

$$
\begin{aligned}
& \epsilon_{1}=\rho^{T} \exp \left\{-r q_{m}-T \gamma\right\}\left(1-\alpha_{1}^{T}\right), \\
& \epsilon_{2}=\rho^{\mathfrak{T}} \exp \left\{-r \mathfrak{q}_{m}-\mathfrak{T} \lambda\right\}\left(1-\alpha_{2}^{\mathfrak{T}}\right) .
\end{aligned}
$$

Since $\mathrm{V}_{T+1}^{x}=\mathrm{V}^{x}, \mathrm{~V}_{\mathfrak{T}+1}^{y}=\mathrm{V}^{y}$, we have $\Delta_{T+1}=E_{T+1}, \nabla_{\mathfrak{T}+1}=R_{\mathfrak{T}+1}$, hence (3.25), (3.26) imply

$$
\begin{aligned}
& \tilde{E}_{T+1} \leq \epsilon_{1} \tilde{E}_{1}, \\
& \tilde{R}_{\mathfrak{T}+1} \leq \epsilon_{2} \tilde{R}_{1} .
\end{aligned}
$$

Now, we get the relationships of $\tilde{E}_{T+1}$ and $\tilde{E}_{1}, \tilde{R}_{\mathfrak{T}+1}$ and $\tilde{R}_{1}$, So when $m=1$, similar to the analysis of (3.25) and (3.26), we can obtain

$$
\begin{aligned}
& \tilde{E}_{2 T+1} \leq \epsilon_{1} \tilde{E}_{T+1}, \\
& \tilde{R}_{2 \mathfrak{T}+1} \leq \epsilon_{2} \tilde{R}_{\mathfrak{T}+1} .
\end{aligned}
$$

By iterations, we can get

$$
\begin{aligned}
& \tilde{E}_{m T+1} \leq \epsilon_{1} \tilde{E}_{(m-1) T+1} \leq \cdots \leq \epsilon_{1}^{m} \tilde{E}_{1}, \\
& \tilde{R}_{m \mathfrak{T}+1} \leq \epsilon_{2}^{1} \tilde{R}_{(m-1) \mathfrak{T}+1} \leq \cdots \leq \epsilon_{2}^{m} \tilde{R}_{1} .
\end{aligned}
$$

Hence, $\lim _{m \rightarrow \infty} \tilde{E}_{m T+1}=0$ and $\lim _{m \rightarrow \infty} \tilde{R}_{m T+1}=0$, which means $\lim _{t \rightarrow \infty} \max \| x_{i}(t)-$ $x_{j}(t) \|=0, i, j \in \mathrm{V}^{x}$ and $\lim _{t \rightarrow \infty} \max \left\|y_{i}(t)-y_{j}(t)\right\|=0, i, j \in \mathrm{V}^{y}$ then MNNs (2.9) and (2.10) are in global synchronization.

Remark 3.1 The NNs models in this paper are slightly distinct from the general NNs model. By observing the models (2.9) and (2.10) we find that the two networks are not only affected by the impulsive coupling control of their own sub-networks, but also each node is affected by the corresponding node of another MNNs. Compared with other network models, this model has more complex dynamic behavior. In addition, impulsive coupling control is used in this paper, which saves communication resources more than continuous coupling.

Remark 3.2 Theorem 3.1 offers a sufficient criterion under which global synchronization of MNNs models (2.9) and (2.10) can be achieved with conditions (1), (2) and Assumption 1 . How to make the sufficient conditions more general present challenges for future research.

Remark 3.3 The impulsive sequence in this paper is a set of given random sequences, but in order to achieve global synchronization, not all impulsive instants need to be coupled, 
so the number of control can be further reduced. In the existing methods, we can use an event-triggered scheme to further reduce the number of impulsive sequences, but how to design this event-triggered scheme to work more efficiently is also a direction we will consider in our next work.

Remark 3.4 For proving the synchronization of general networks, we mainly prove the stability of the error system which is composed of a drive system and a response system. However, the synchronization of MNNs requires the trajectory of all nodes to converge to one track. In this paper, we define two convex hulls $\Delta(t)$ and $\nabla(t)$, which introduced in Sect. 2.3 (the definition of the convex hull of $M$ ), then the diameter of $\Delta(t)$ and $\nabla(t)$ means the maximum error between nodes, when the diameter of the two convex hulls tends to zero, the maximum error between the nodes tends to zero, and this means that the two MNNs are synchronized.

Remark 3.5 The main proof ideas of this paper can be summed up as follows: Firstly, we give the relation between the error of impulsive instant $t_{k}^{+}\left(\tilde{e}_{i j}\left(t_{k}^{+}\right)\right)$and the error of nonimpulsive instant $t \in\left(t_{k}, t_{k+1}\right]\left(e_{i j}(t)\right)$ by Lemma 2.1, then we get the relationship $\| x_{i}(t)-$ $x_{j}(t)\left\|i, j \in \mathrm{V}^{x},\right\| y_{i}(t)-y_{j}(t) \| i, j \in \mathrm{V}^{y}$ between the maximum error at impulsive instant $t_{k}$ and at $t \in\left(t_{k}, t_{k+1}\right]$, respectively. In other words, we get the relationships of $\Delta_{k}, \Delta_{k}^{+}$and $\nabla_{k}$, $\nabla_{k}^{+}$. Next, we derive the expansion rates of $\Delta(t)$ and $\nabla(t)$ in an impulsive interval $\left(t_{k}, t_{k+1}\right]$, and $\Delta(t), \nabla(t)$ will be reduced by impulsive coupling. By conditions (2.15), (2.16), we can see that the decreasing rates of $\Delta(t)$ and $\nabla(t)$ are larger than their expansion rates, then the two MNNs are able to achieve synchronization.

\section{Numerical example}

In this section, in order to prove the validity of the appeal theorem, we give the following numerical simulation.

Example 4.1 Consider the MNNs (2.9) and (2.10) with $N=4$

$$
\begin{aligned}
& \left\{\begin{array}{l}
\frac{d x_{i}(t)}{d t}=-C x_{i}(t)+A f\left(y_{j}(t)\right)+B f^{\tau}\left(y_{j}\left(t-\tau_{1}\right)\right)+I(t), \quad t \neq t_{k}, \\
x_{i}\left(t_{k}^{+}\right)=\sum_{j=1}^{N} d_{i j}\left(t_{k}\right) \Gamma\left(t_{k}\right) x_{j}\left(t_{k}\right),
\end{array}\right. \\
& \left\{\begin{array}{l}
\frac{d y_{j}(t)}{d t}=-\mathfrak{C} y_{j}(t)+\mathfrak{A} g\left(x_{i}(t)\right)+\mathfrak{B} g^{\tau}\left(x_{i}\left(t-\tau_{2}\right)\right)+\mathfrak{I}(t), \quad t \neq t_{k}, \\
y_{j}\left(t_{k}^{+}\right)=\sum_{i=1}^{N} \mathfrak{d}_{j i}\left(t_{k}\right) \Lambda\left(t_{k}\right) y_{i}\left(t_{k}\right),
\end{array}\right.
\end{aligned}
$$

where $x_{i}(t)=\left(x_{i 1}(t), x_{i 2}(t)\right)^{T}, y_{j}(t)=\left(y_{j 1}(t), y_{j 2}(t)\right)^{T}$ are the state vectors. It is assumed that $f(x)=f^{\tau}(x)=\left(f_{1}\left(x_{1}\right), f_{2}\left(x_{2}\right)\right)^{T}, g(y)=g^{\tau}(y)=\left(g_{1}\left(y_{1}\right), g_{2}\left(y_{2}\right)\right)^{T}$, and $f_{i}\left(x_{i}\right)=(1 / 2)\left(\left|x_{i}+1\right|-\mid x_{i}-\right.$ $1 \mid), i=1,2, g_{j}\left(y_{j}\right)=(1 / 3)\left(\left|y_{j}+1\right|-\left|y_{j}-1\right|\right), j=1,2$. The delays of $\tau_{1}$ and $\tau_{2}$ are set to be 0.1 . We give the initial states by $x_{11}(s)=0.2, x_{12}(s)=0.5, x_{21}(s)=0.1, x_{22}(s)=0.65, x_{31}(s)=-0.35$, $x_{32}(s)=-3, x_{41}(s)=-0.7, x_{42}(s)=-0.6, y_{11}(s)=0.2, y_{12}(s)=0.3, y_{21}(s)=0.4, y_{22}(s)=0.15$, $y_{31}(s)=-0.5, y_{32}(s)=-0.8, y_{41}(s)=-0.45, y_{42}(s)=-0.1, s \in[-0.1,0]$.

Taking

$$
C=\left[\begin{array}{cc}
1.5 & 0 \\
0 & 1.5
\end{array}\right], \quad A=\left[\begin{array}{cc}
\frac{\sqrt{2}}{10} & 10 \\
0.1 & \frac{\sqrt{2}}{10}
\end{array}\right], \quad B=\left[\begin{array}{cc}
-\frac{3 \sqrt{2} \pi}{40} & 0.2 \\
0.2 & -\frac{3 \sqrt{2} \pi}{40}
\end{array}\right],
$$




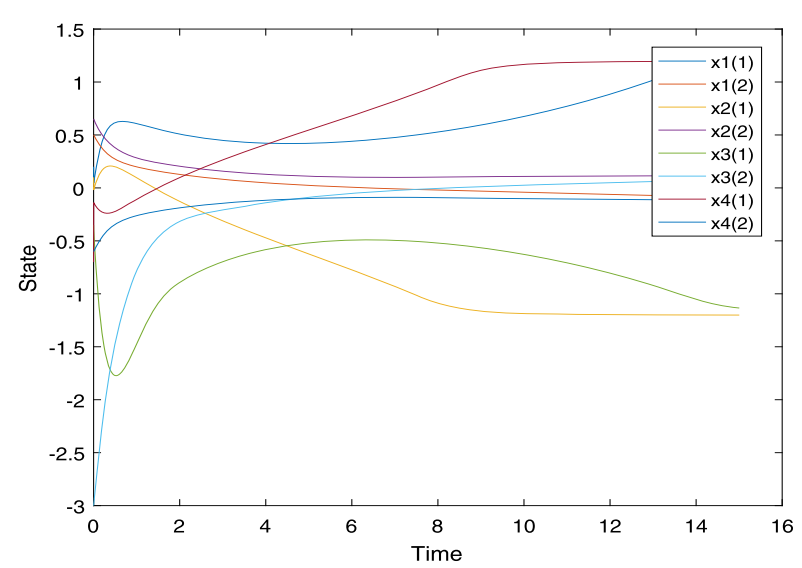

Figure 1 State trajectory of (4.1) without control

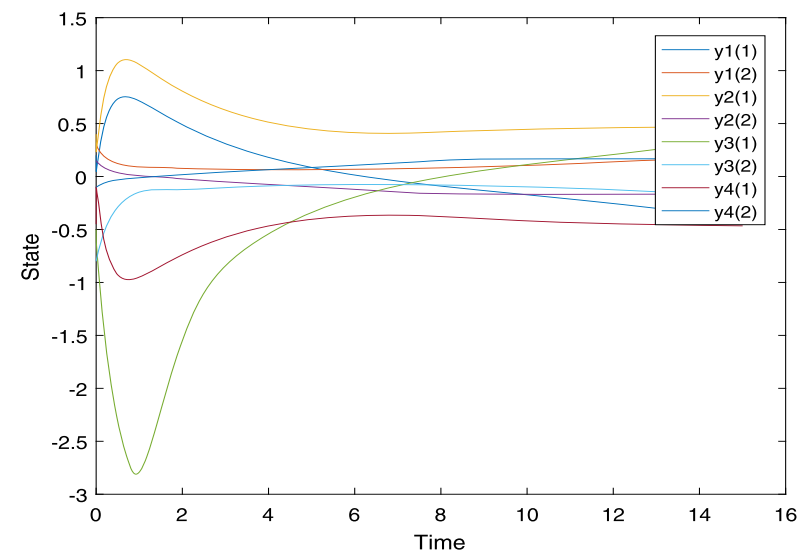

Figure 2 State trajectory of (4.2) without control

$$
\mathfrak{C}=\left[\begin{array}{cc}
2 & 0 \\
0 & 2.5
\end{array}\right], \quad \mathfrak{A}=\left[\begin{array}{cc}
\frac{\sqrt{2} \pi}{20} & 10 \\
0.5 & \frac{\sqrt{2} \pi}{20}
\end{array}\right], \quad \mathfrak{B}=\left[\begin{array}{cc}
-\frac{4+\pi}{15} & 0.1 \\
0.1 & -\frac{4+\pi}{15}
\end{array}\right]
$$

from Figs. 1, 2, 3, and 4, we can see that, if we have MNNs (4.1), (4.2) without the impulsive coupling controls, then the synchronizations cannot be obtained.

Next, consider the switching topology shown in Figs. 5 and 6 connected by choosing $V_{1}^{x}=\{1\}, V_{2}^{x}=\{1,2,3\}, V_{3}^{x}=\{1,2,3,4\}$ and $V_{1}^{y}=\{1\}, V_{2}^{y}=\{1,2,3\}, V_{3}^{x}=\{1,2,3,4\}$. We can show that $\left\{\mathrm{G}_{2 m+1}^{x}, \mathrm{G}_{2(m+1)}^{x}\right\}$ and $\left\{\mathrm{G}_{2 m+1}^{y}, \mathrm{G}_{2(m+1)}^{y}\right\}$ are sequentially connected (also jointly connected). The matrices corresponding to the graphs are given as

$$
D\left(t_{2 m+1}\right)=\left[\begin{array}{cccc}
1 & 0 & 0 & 0 \\
0.5 & 0.5 & 0 & 0 \\
0.7 & 0 & 0.3 & 0 \\
0 & 0 & 0 & 1
\end{array}\right], \quad D\left(t_{2(m+1)}\right)=\left[\begin{array}{cccc}
0.7 & 0 & 0 & 0.3 \\
0 & 1 & 0 & 0 \\
0 & 0 & 1 & 0 \\
0 & 0 & 0.8 & 0.2
\end{array}\right],
$$




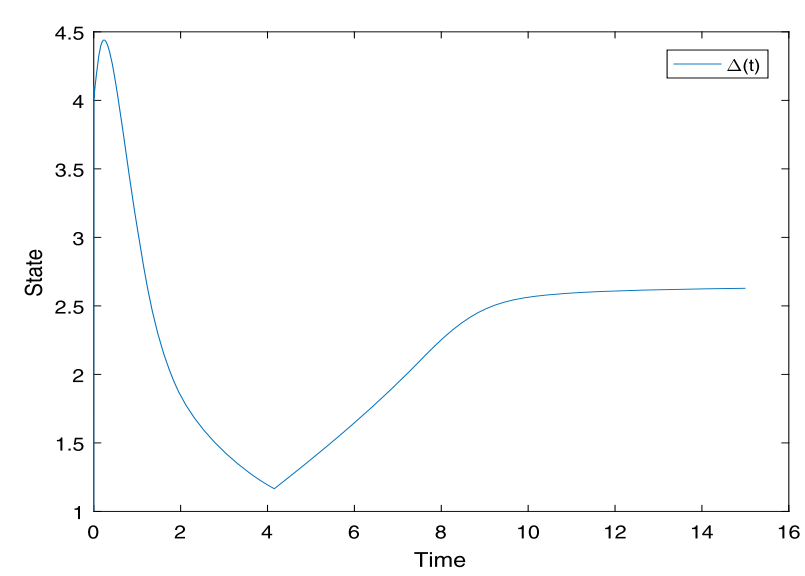

Figure 3 Variation of $\Delta(t)$ in Example 4.1 of (4.1) without control

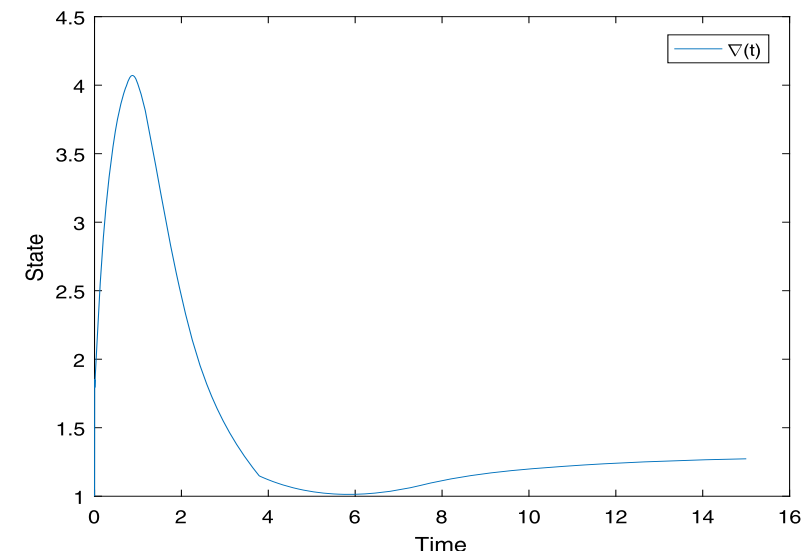

Figure 4 Variation of $\nabla(t)$ in Example 4.1 of (4.2) without control

$$
\mathfrak{D}\left(t_{2 m+1}\right)=\left[\begin{array}{cccc}
1 & 0 & 0 & 0 \\
0 & 0.8 & 0.2 & 0 \\
0.9 & 0 & 0.1 & 0 \\
0 & 0 & 0 & 1
\end{array}\right], \quad \mathfrak{D}\left(t_{2(m+1)}\right)=\left[\begin{array}{cccc}
0.8 & 0 & 0 & 0.2 \\
0 & 1 & 0 & 0 \\
0 & 0 & 1 & 0 \\
0 & 0.4 & 0 & 0.6
\end{array}\right] .
$$

Noting that $d_{i 1}\left(t_{2 m+1}\right) \geq 0.5, \mathfrak{d}_{i 1}\left(t_{2 m+1}\right) \geq 0.9$ for any $i \in \mathrm{V}_{2}^{x}$ and $i \in \mathrm{V}_{2}^{y}$. Also we have $\sum_{j \in \mathrm{V}_{2}^{x}} d_{i j}\left(t_{2(m+1)}\right)=1, \sum_{j \in \vee_{2}^{y}} \mathfrak{d}_{i j}\left(t_{2(m+1)}\right)=1$ for any $i \in \mathrm{V}_{3}^{x}, i \in \mathrm{V}_{3}^{y}$, then let $\alpha_{1}=0.5, \alpha_{2}=0.9$. From conditions (2.13) and (2.14) of Lemma 2.1, we solve the following inequality groups by MATLAB:

$$
\begin{aligned}
& r-c_{\min }+\|A P\|+\left\|B P^{\tau}\right\| \exp \{r \tau\}=r-1.5+5.0707+0.2666 \times \exp \{r \tau\} \leq 0, \\
& r-\mathfrak{c}_{\min }+\|\mathfrak{A} L\|+\left\|\mathfrak{B} L^{\tau}\right\| \exp \{r \tau\}=r-2.5+3.4074+0.1920 \times \exp \{r \tau\} \leq 0,
\end{aligned}
$$

then we can get $r<=-1.0797$, choose $r=-6$, then $\rho=\max \{1, \exp \{-6 \times 0.1\}\}=1$. Let $q_{m}=$ $\mathfrak{q}_{m}=0.6$, then the impulsive sequence satisfies $t_{2(m+1)}-t_{2 m+1}=0.3$. In order to get the 


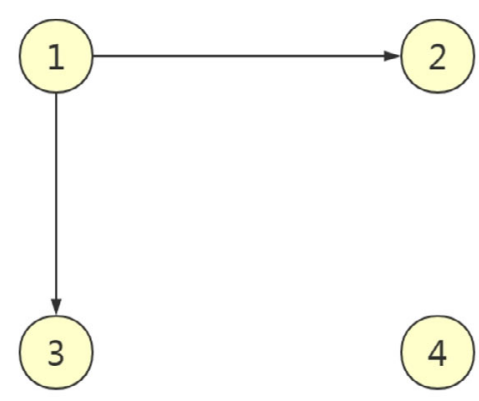

(a) $t=t_{2 m+1}$

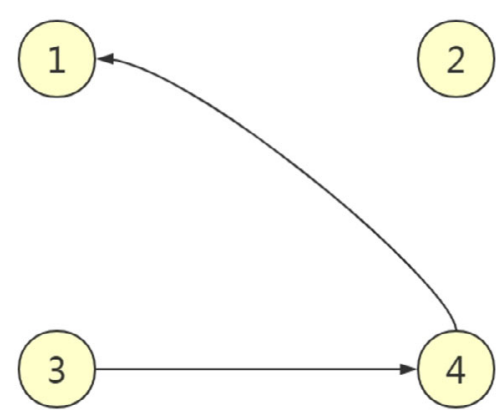

(b) $t=t_{2(m+1)}$

Figure 5 Communication topology among four NNs of $\mathrm{G}^{x}, m \in Z^{+}$

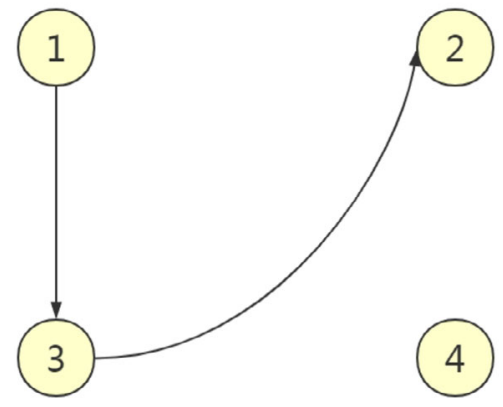

(a) $t=t_{2 m+1}$

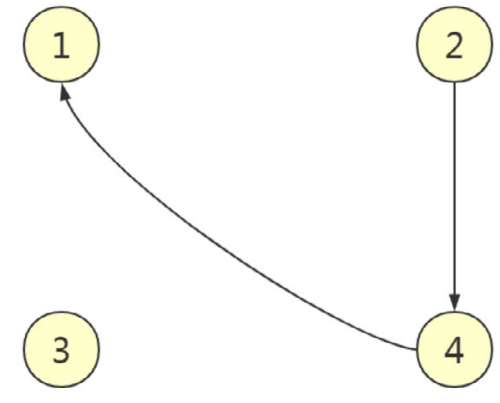

(b) $t=t_{2(m+1)}$

Figure 6 Communication topology among four NNs of $\mathrm{G}^{y}, m \in Z^{+}$

conditions (3.3), (3.4) of Theorem 3.1, then solve the following inequality groups:

$$
\begin{array}{ll}
\rho^{T} \exp \left\{-r q_{m}-T \gamma\right\}\left(1-\left(\alpha_{1}\right)^{T}\right) & =1 \times \exp \{-6 \times 0.6-0.6 \times \gamma\}\left(1-0.5^{0.6}\right)<1, \\
\rho^{\mathfrak{T}} \exp \left\{-r \mathfrak{q}_{m}-\mathfrak{T} \lambda\right\}\left(1-\left(\alpha_{2}\right)^{\mathfrak{T}}\right) & =1 \times \exp \{-6 \times 0.6-0.6 \times \lambda\}\left(1-0.9^{0.6}\right)<1,
\end{array}
$$

we can get $\gamma>-7.7969, \lambda>-10.6544$, let $\gamma=-7, \lambda=-10$, then by the definitions $\gamma_{\max }=$ $\max _{k \geq 1, i \in N}\left\{\gamma_{i}\left(t_{k}\right)\right\}, \lambda_{\max }=\max _{k \geq 1, i \in N}\left\{\lambda_{i}\left(t_{k}\right)\right\}$ and $\gamma=-\ln \left(\gamma_{\max }\right), \lambda=-\ln \left(\lambda_{\max }\right)$, we can get $\gamma_{\max }=\exp \{7\}, \lambda_{\max }=\exp \{10\}$, then the coupling gains relative to the switching topologies of $\left\{\mathrm{G}^{x}\right\}$ and $\left\{\mathrm{G}^{y}\right\}$ are given by

$$
\begin{array}{rlr}
\Gamma\left(t_{2 m+1}\right)=\left[\begin{array}{cc}
-0.8 & 0 \\
0 & 0.0029
\end{array}\right], & \Gamma\left(t_{2(m+1)}\right)=\left[\begin{array}{cc}
-0.8 & 0 \\
0 & 0.0029
\end{array}\right], \\
\Lambda\left(t_{2 m+1}\right)=\left[\begin{array}{cc}
-0.8 & 0 \\
0 & 0.0057
\end{array}\right], & \Lambda\left(t_{2(m+1)}\right)=\left[\begin{array}{cc}
-0.8 & 0 \\
0 & 0.0056
\end{array}\right],
\end{array}
$$

then the conditions (1), (2) of Theorem 3.1 are satisfied, MNNs (4.1) and (4.2) can reach global synchronizations with impulsive coupling controls under switching topologies of Figs. 5 and 6. The state trajectories of all MNNs are illustrated in Figs. 7 and 8. We can see that the two MNNs achieve synchronizations finally. Moreover, the max state errors 


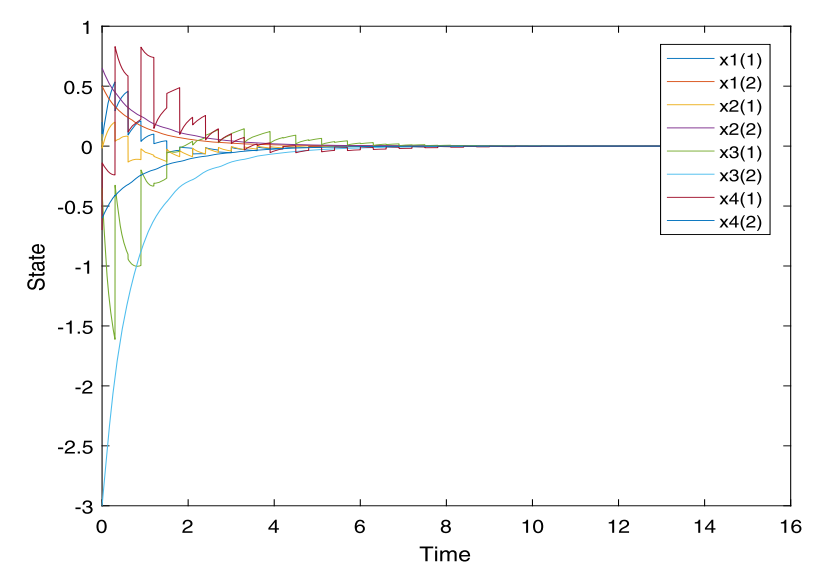

Figure 7 State trajectory of (4.1) with control

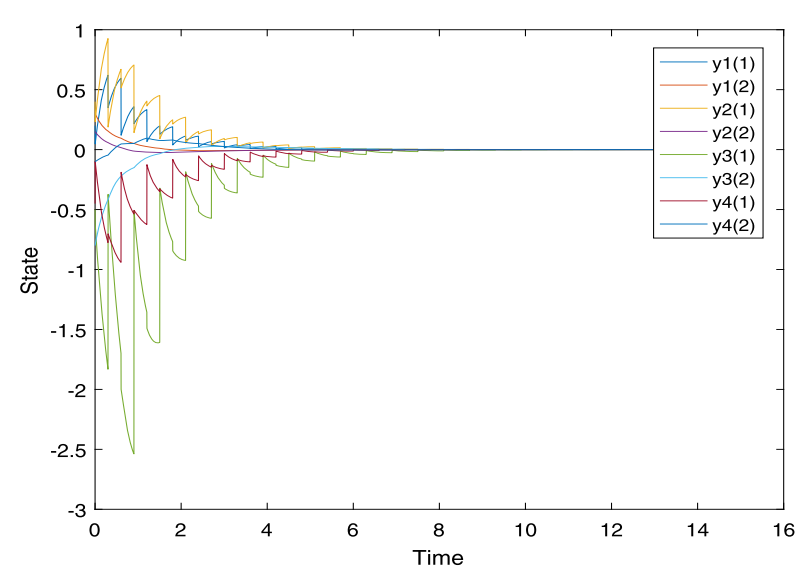

Figure 8 State trajectory of (4.2) with control

between four NNs of (4.1) and (4.2) are defined as

$$
\begin{aligned}
& \Delta(t)=\max _{1 \leq i, j \leq 4}\left\|x_{i}(t)-x_{j}(t)\right\|, \\
& \nabla(t)=\max _{1 \leq i, j \leq 4}\left\|y_{i}(t)-y_{j}(t)\right\| .
\end{aligned}
$$

Then $\Delta(t) \rightarrow 0$ and $\nabla(t) \rightarrow 0$ as $t \rightarrow \infty$, which are shown in Figs. 9 and 10.

\section{Conclusion}

This paper discusses the global synchronizations of the two interconnected bidirection MNNs with time-delay via an impulsive coupling controller in which the coupling topology is variable. After satisfying certain conditions, the two MNNs which affect each other and achieve synchronizations under the action of impulsive coupling. Compared with continuous coupling, impulsive coupling can reduce communication bandwidth. In addition, we give the principle that the model can achieve synchronization under the impulsive coupling controller. Each of the two networks in this paper is not only affected by impulsive 


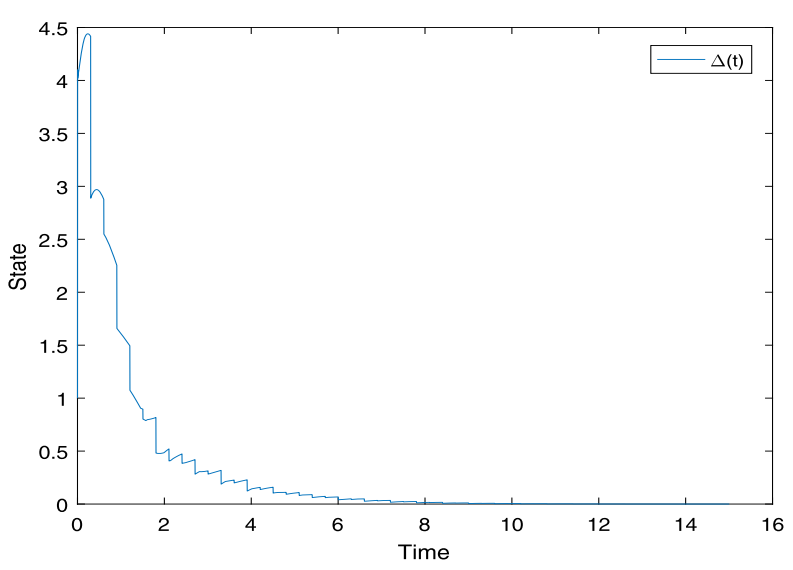

Figure 9 Variation of $\Delta(t)$ in Example 4.1 of (4.1) with control

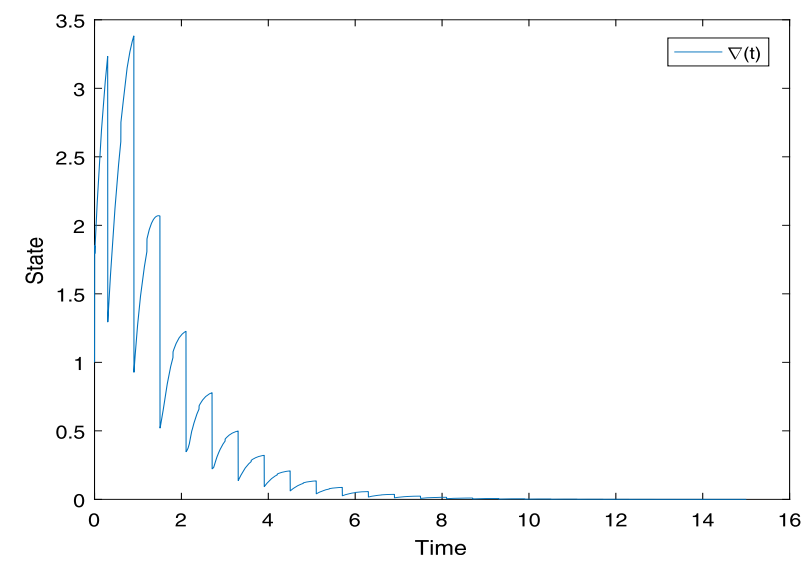

Figure 10 Variation of $\nabla(t)$ in Example 4.1 of (4.2) with control

coupling, but also affected by the corresponding nodes in another network. The present studies on this kind of problem are few, so the research in this paper has some significance. At the same time, as described in Remark 3.3, the communication data can be further reduced by designing a suitable event-triggered scheme to determine the impulse sequence, this will be considered in the future.

Acknowledgements

The authors are thankful to the editor and the anonymous reviewers for their valuable comments and suggestions.

Funding

This work is supported by the Natural Science Foundation of China under Grants 61976084 and 61773152.

Availability of data and materials

No availability of data and material.

Competing interests

The authors declare that they have no competing interests.

Authors' contributions

All authors contributed equally to this work. All authors read and approved the final manuscript. 


\section{Author details}

${ }^{1}$ College of Applied Mathematics, Hubei Normal University, Huangshi, 435002, China. ${ }^{2}$ College of Mathematics and Statistics, Hubei Normal University, Huangshi, 435002, China.

\section{Publisher's Note}

Springer Nature remains neutral with regard to jurisdictional claims in published maps and institutional affiliations.

Received: 5 February 2020 Accepted: 22 June 2020 Published online: 06 July 2020

\section{References}

1. Mohanty, R., Mallik, B.K., Solanki, S.S.: Automatic bird species recognition system using neural network based on spike. Appl. Acoust. 161, Article ID 107117 (2020)

2. Ali, S., Zhang, J.R., Azeem, A., Mahmood, A.: Impact of electricity consumption on economic growth: an application of vector error correction model and artificial neural networks. J. Dev. Areas 54(4), 90-104 (2020)

3. He, C., Li, J.M.: Event-based aperiodically intermittent pinning synchronization control strategy for linearly coupled complex networks. Nonlinear Anal. Hybrid Syst. 36, Article ID 100836 (2020)

4. Pikovsky, A., Rosenblum, M., Kurths, J.: Synchronization: A Universal Concept in Nonlinear Sciences, vol. 12. Cambridge University Press, Cambridge (2003)

5. Ramirez, J.P., Garcia, E., Alvarez, J.: Master-slave synchronization via dynamic control. Commun. Nonlinear Sci. Numer. Simul. 80(2020), Article ID 104977 (2019)

6. He, W., Qian, F., Cao, J.: Pinning-controlled synchronization of delayed neural networks with distributed-delay coupling via impulsive control. Neural Netw. 85, 1-9 (2017)

7. Fečkan, M., Sathiyaraj, T., Wang, J.R.: Synchronization of butterfly fractional order chaotic system. Mathematics 8(3), $446(2020)$

8. Alsaedi, A., Usha, M., Syed Ali, M., Ahmad, B.: Finite-time synchronization of sampled-data Markovian jump complex dynamical networks with additive time-varying delays based on dissipative theory. J. Comput. Appl. Math. 368 Article ID 112578 (2020)

9. Martínez-Guerra, R., Cruz-Ancona, C.D., Pérez-Pinacho, C.A.: Generalized multi-synchronization viewed as a multi-agent leader-following consensus problem. Appl. Math. Comput. 282, 226-236 (2016)

10. Cruz-Ancona, C.D., Martínez-Guerra, R., Pérez-Pinacho, C.A.: Generalized multi-synchronization: a leader-following consensus problem of multi-agent systems. Neurocomputing 233, 52-60 (2017)

11. Shen, B., Wang, Z., Liu, X.: Sampled-data synchronization control of dynamical networks with stochastic sampling. IEEE Trans. Autom. Control 57(10), 2644-2650 (2012)

12. Yang, S.F., Guo, Z.Y., Wang, J.: Robust synchronization of multiple memristive neural networks with uncertain parameters via nonlinear coupling. IEEE Trans. Syst. Man Cybern. Syst. 45(7), 1077-1086 (2015)

13. Lu, W.L., Chen, T.P.: Synchronization of coupled connected neural networks with delays. IEEE Trans. Circuits Syst. I, Regul. Pap. 51(12), 2491-2503 (2004)

14. Liu, Y.C., Liao, X.F.: FLS-based finite-time synchronization of delayed memristive neural networks with interval parameters and nonlinear couplings. Phys. A, Stat. Mech. Appl. 553, Article ID 121890 (2019)

15. Wang, W.W., Cao, J.D.: Synchronization in an array of linearly coupled networks with time-varying delay. Phys. A, Stat. Mech. Appl. 336(1), 197-211 (2006)

16. Mathiyalagan, K., Park, J.H., Sakthivel, R.: Synchronization for delayed memristive BAM neural networks using impulsive control with random nonlinearities. Appl. Math. Comput. 259(15), 967-979 (2015)

17. Li, N., Cao, J.D.: Synchronization criteria for multiple memristor-based neural networks with time delay and inertial term. Sci. China, Technol. Sci. 61(4), 612-622 (2018)

18. Yang, S.F., Guo, Z.Y., Wang, J.: Global synchronization of multiple recurrent neural networks with time delays via impulsive interactions. IEEE Trans. Neural Netw. Learn. Syst. 28(7), 1657-1667 (2017)

19. Chen, J.J., Zeng, Z.G., Chen, B.S.: Effects of subsystem and coupling on synchronization of multiple neural networks with delays via impulsive coupling. IEEE Trans. Neural Netw. Learn. Syst. 30(12), 3748-3758 (2019)

20. Chen, Y., Yu, W., Li, F., Feng, S.: Synchronization of complex networks with impulsive control and disconnected topology. IEEE Trans. Circuits Syst. II, Express Briefs 60(5), 292-296 (2013)

21. Jia, Y., Wu, H.Q., Cao, J.D.: Non-fragile robust finite-time synchronization for fractional-order discontinuous complex networks with multi-weights and uncertain couplings under asynchronous switching. Appl. Math. Comput. 370, Article ID 124929 (2020)

22. Cheng, W.K., Wu, A.L., Zhang, J.E., Li, B.L.: Adaptive control of Mittag-Leffler stabilization and synchronization for delayed fractional-order BAM neural networks. Adv. Differ. Equ. 2019, Article ID 337 (2019)

23. Li, L., Chen, W.S., Wu, X.J.: Global exponential stability and synchronization for a novel complex-valued neural networks with proportional delays and inhibitory factors. IEEE Trans. Cybern. https://doi.org/10.1109/TCYB.2019.2946076

24. Li, X.D., Martin, B.: An impulsive delay differential inequality and applications. Comput. Math. Appl. 64(6), 1875-1881 (2012)

25. Li, X.D., Shen, J.H., Rakkiyappan, R.: Persistent impulsive effects on stability of functional differential equations with finite or infinite delay. Appl. Math. Comput. 329, 14-22 (2018)

26. Li, X.D., Yang, X.Y., Hang, T.W.: Persistence of delayed cooperative models: impulsive control method. Appl. Math Comput 342, 130-146 (2019) 